\title{
Cascaded orthogonal space-time block codes for wireless multi-hop relay networks
}

\author{
Rahul Vaze ${ }^{1 *}$ and Robert W Heath Jr.2
}

\begin{abstract}
Distributed space-time block coding is a diversity technique to mitigate the effects of fading in multi-hop wireless networks, where multiple relay stages are used by a source to communicate with its destination. This article proposes a new distributed space-time block code called the cascaded orthogonal space-time block code (COSTBC) for the case where the source and destination are equipped with multiple antennas and each relay stage has one or more multiple antenna relays. Each relay stage is assumed to have receive channel state information (CSI) for all the channels from the source and all relays from previous stages to itself, while the destination is assumed to have receive CSI for all the channels. To construct the COSTBC, multiple orthogonal space-time block codes (OSTBCs) are used in cascade by the source and each relay stages. In the COSTBC, each relay stage separates the constellation symbols of the OSTBC sent by the preceding relay stage using its CSI, and then transmits another OSTBC to the next relay stage. COSTBCs are shown to achieve the maximum diversity gain in a multi-hop wireless network with linear decoding complexity thanks to the connection to OSTBCs. Several explicit constructions of COSTBCs are also provided, and their performance is simulated in different relay configurations.
\end{abstract}

\section{Introduction}

Distributed space-time block coding (DSTBC) is a technique to improve reliability in relay-assisted communication, where one or more relays help the source to communicate with its destination. Relay-assisted communication is likely to occur in large wireless networks, such as ad-hoc or sensor network, where the destination is possibly out of the source's communication range. Relay-assisted communication is also used in a cellular wireless networks to improve the performance of cell edge users, and has been incorporated in modern wireless standards such as IEEE 802.16j, and 3GPP LTE Advanced.

In DSTBCs, relay antennas are used together with the source antennas in a distributed manner to transmit a space-time block code (STBC) [1] to the destination. By introducing redundancy in space and time, DSTBCs increase the reliability of the communication by increasing the diversity gain, defined as the negative of the exponent of the signal-to-noise ratio (SNR) in the pairwise error probability expression at high SNR [1].

\footnotetext{
*Correspondence: vaze@tcs.tifr.res.in

1 School of Technology and Computer Science, Tata Institute of Fundamental Research, Mumbai 400005, India

Full list of author information is available at the end of the article
}

In prior work, maximum diversity gain achieving DSTBC constructions have been proposed for the twohop network [2-21], and for the multi-hop network [22-24]. Even though these DSTBC constructions [2-24] achieve the maximum diversity gain, the decoding complexity of most of them, except [14-21], is very high, thereby limiting their use in practical deployment. Construction of DSTBCs with low decoding complexity is practically important as highlighted by the fact that the Alamouti code is the most practically used code not only because it achieves the maximum diversity gain, but also because it requires minimum decoding complexity. Moreover, the DSTBC constructions with low decoding complexity [14-21] are limited to two-hop network with single antenna equipped source, destination, and the relay nodes.

In this article, we design maximum diversity gain achieving DSTBCs with low-decoding complexity for a multi-hop wireless network where the source, the destination, and the relay nodes are equipped with multiple antennas. In the proposed DSTBC, called the cascaded orthogonal space-time block code (COSTBC), an orthogonal space-time code (OSTBC) [25] is used by the source, and subsequently by each relay stage to communicate with its adjacent relay stage. OSTBCs are considered because

\section{Springer}

(c) 2013 Vaze and Heath; licensee Springer. This is an Open Access article distributed under the terms of the Creative Commons Attribution License (http://creativecommons.org/licenses/by/2.0), which permits unrestricted use, distribution, and reproduction in any medium, provided the original work is properly cited. 
of their single symbol decodable property $[25,26]$, i.e., with the maximum likelihood decoding each constellation symbol of the OSTBC can be decoded independently of other constellation symbols. We assume that each relay has receive channel state information (CSI) for all the channels from the source to itself, while the destination is assumed to have receive CSI for all the channels. With COSTBCs, in the first time slot, the source transmits an OSTBC to the first relay stage. Using the orthogonality property of the OSTBC and the available CSI, each relay of the first relay stage separates the different OSTBC constellation symbols from the received signal, and transmits a codeword vector in the next time slot, such that the matrix obtained by stacking all the codeword vectors transmitted by the different relays of the first relay stage is an OSTBC. These operations are repeated by subsequent relay stages. With COSTBCs, no signal is decoded at any of the relays, therefore COSTBC construction with single antenna relays is equivalent to COSTBC construction with multiple antenna relays. Thus, without loss of generality, in this article, we only consider COSTBC construction for single antenna relays. We note that for the code construction each relay is required to have receive CSI for all the channels from the source and all relays from previous stages to itself, while the destination is assumed to have receive CSI for all the channels.

\subsection{Our contributions}

- We show that COSTBCs achieve the maximum diversity gain in a multi-hop wireless network when each symbol of the code is decoded independently (non- maximum-likelihood decoding), resulting in linear decoding complexity similar to single symbol decodable codes.

- We prove that for a two-hop network and when the destination has a single antenna, by adding channel coefficient-dependent noise terms to the received signals, COSTBCs have the single symbol decodable property for any number of source and relay antennas. Thus, by paying a penalty in terms of coding gain because of extra noise, COSTBCs provide significant decoding complexity gain.

A part of this article has been presented at $[27,28]$. Due to space limitation, the studies $[27,28]$ contain only the results of this article without any proofs. In this article, detailed proofs of the results, together with explicit code construction, and some simulation results are described.

\subsection{Comparison with prior work}

Previous constructions of maximum diversity gain achieving DSTBCs with low decoding complexity (single symbol decodable) [14-21] are limited to a two-hop network with single antenna nodes. COSTBCs, in comparison, achieve the maximum diversity gain with linear decoding complexity (similar to [14-21]) in a multi-hop network with multiple antenna equipped nodes, even though they do not have the single symbol decodable property. For the multi-hop network, the focus of $[23,24]$ is on the construction of DSTBCs that can achieve the optimal diversity multiplexing tradeoff [29]. In comparison to the strategies of $[23,24]$, COSTBCs only achieve the maximum diversity gain and fall short of achieving the maximum multiplexing gain because of the use of OSTBCs. The decoding complexity of COSTBC, however, is significantly less (linear) than the strategies of $[23,24]$ and makes COSTBCs amenable for practical implementation in comparison to $[23,24]$, where STBCs with high decoding complexity are used. Thus, COSTBCs are well suited for relay-assisted communication where relays are used to improve the cell coverage, by improving reliability of the users at the cell edge, while requiring low decoding complexity.

Notation: Let A denote a matrix, a a vector and $a_{i}$ the $i$ th element of $\mathbf{a}$. $\operatorname{diag}(\mathbf{A})$ represents a vector consisting of diagonal entries of $\mathbf{A}$. The determinant and trace of matrix $\mathbf{A}$ are denoted by $\operatorname{det}(\mathbf{A})$ and $\operatorname{tr}(\mathbf{A})$. The vector consisting of the diagonal entries of $\mathbf{A}$ is denoted by $\operatorname{diag}(\mathbf{A})$. The field of real and complex numbers are denoted by $\mathbb{R}$ and $\mathbb{C}$, respectively. The space of $M \times N$ matrices with complex entries is denoted by $\mathbb{C}^{M \times N}$. The Euclidean norm of a vector $\mathbf{a}$ is denoted by $|\mathbf{a}|$. An $m \times m$ identity matrix is denoted by $\mathbf{I}_{m}$, and $\mathbf{0}_{m}$ is as an all zero $m \times m$ matrix. The superscripts ${ }^{T},{ }^{*},{ }^{\dagger}$ represent the transpose, transpose conjugate, and element wise conjugate. The expectation of function $f(x)$ with respect to $x$ is denoted by $\mathbb{E}\{f(x)\}$. A circularly symmetric complex Gaussian random variable $x$ with zero mean and variance $\sigma^{2}$ is denoted as $x \sim \mathcal{C} N\left(0, \sigma^{2}\right)$. We use the symbol $\doteq$ to represent exponential equality, i.e., let $f(x)$ be a function of $x$, then $f(x) \doteq x^{a}$ if $\lim _{x \rightarrow \infty} \frac{\log (f(x))}{\log x}=a$ and similarly $\leq$ and $\geq$ denote the exponential less than or equal to and greater than or equal to relation, respectively. We use the symbol := to define a variable.

\section{System model}

Consider a multi-hop wireless network where a source terminal with $M_{0}$ antennas wants to communicate with a destination terminal with $M_{N}$ antennas via $N-1$ relay stages as shown in Figure 1. We refer to the multi-hop wireless network with $N-1$ relay stages as an $N$-hop network. Each relay in any relay stage has a single antenna; $M_{n}$ denotes the number of relays in the $n$th relay stage. It is assumed that the relays do not generate their own data. Similar to the model considered in [23,24] we assume that any relay of relay stage $n$ can only receive the signal from any relay of relay stage $n-1$, i.e., we consider a directed multi-hop wireless network. This assumption is valid for the case when successive relay stages appear in increasing 


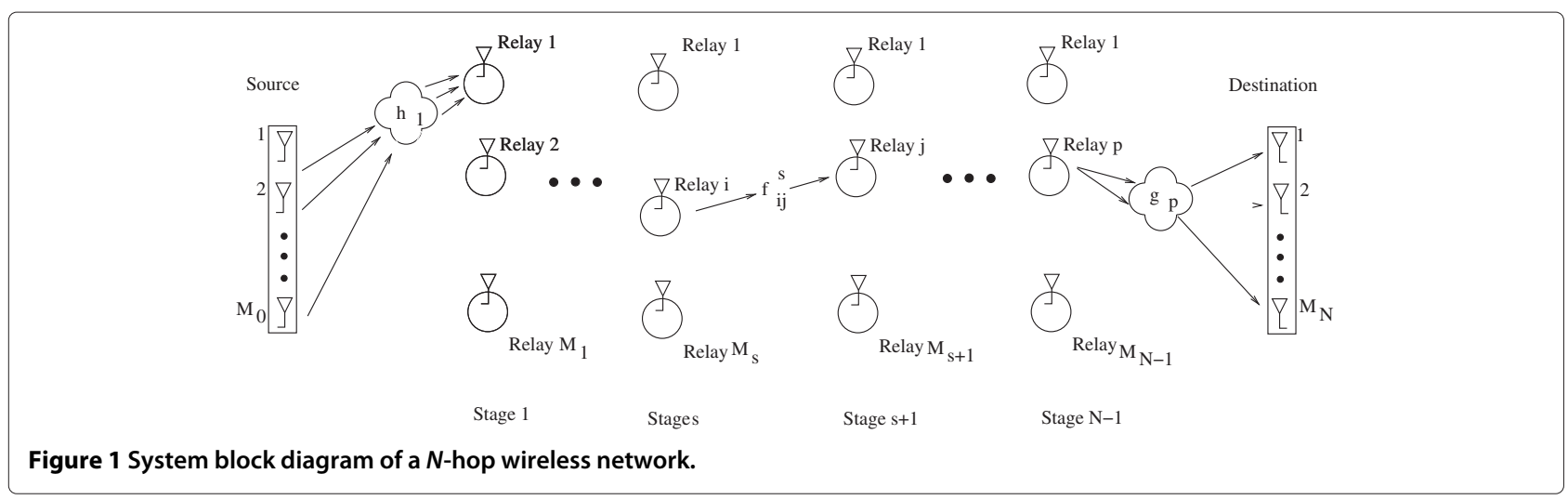

order of distance from the source towards the destination and any two relay nodes are chosen to lie in adjacent relay stages if they have sufficiently good SNR between them. In any practical setting, there will be interference received at any relay node of stage $n$ because of the signals transmitted from relay nodes of relay stage $0, \ldots, n-2$ and $n+2, \ldots, N-1$. Due to relatively large distances between non-adjacent relay stages, however, this interference is quite small and we account for that in the additive noise term similar to [24]. More details are described in Remark 1.

As shown in Figure 1, the channel between the source and the $i$ th relay of the first stage of relays is denoted by $\mathbf{h}_{i}=\left[\begin{array}{llll}h_{1 i} & h_{2 i} & \ldots & h_{M_{0} i}\end{array}\right]^{T}, i=1,2, \ldots, M_{1}$, between the $j$ th relay of relay stage $s$ and the $k$ th relay of relay stage $s+1$ by $f_{j k}^{s}, s=0,1, \ldots, N-2, æ=1,2, \ldots, M_{s}, k=$ $1,2, \ldots, M_{s+1}$ and the channel between the relay stage $N-1$ and the $\ell$ th antenna of the destination by $\mathbf{g}_{\ell}=$ $\left[g_{1 \ell} g_{2 \ell} \ldots g_{M_{N-1} \ell}\right]^{T}, \ell=1,2, \ldots, M_{N}$. We assume that $\mathbf{h}_{i} \in \mathbb{C}^{M_{0} \times 1}, f_{j k}^{s} \in \mathbb{C}^{1 \times 1}, \mathbf{g}_{l} \in \mathbb{C}^{M_{N-1} \times 1}$ are independent and identically distributed (i.i.d.) $\mathcal{C} N(0,1)$ entries for all $i, j, k, \ell, s$. We assume that the $m^{\text {th }}$ relay of $n^{\text {th }}$ stage knows $\mathbf{h}_{i}, f_{j k}^{s}, \forall i, j, k, s=1,2, \ldots, n-2, f_{j m}^{n-1} \forall j$, and the destination knows $\mathbf{h}_{i}, f_{j k}^{s}, \mathbf{g}_{l}, \forall i, j, k, l, s$. We further assume that all these channels are frequency flat and block fading, where the channel coefficients remain constant in a block of time duration $T_{c}$ and change independently from block-to-block. We assume that the $T_{c}$ is at least $\max \left\{M_{0}, M_{1}, \ldots, M_{N-1}\right\}$.

Remark 1. Duplexing: Duplexing is an important consideration in multi-hop relay networks. For example, if relay stage $n$ is receiving the signal from relay stage $n-$ 1 and relay stage $n+1$ is transmitting to relay stage $n+2$ simultaneously, then relay stage $n$ will receive back flow of signals from relay stage $n+1$ that it has already transmitted. Since each relay stage uses an amplify and forward strategy, most of the power at relay stage $n$ will then be used to retransmit signals that have been transmitted before. A related paper [24] claims that back flow can be allowed with successive relay stages transmitting simultaneously without decreasing the diversity gain. That is true, however, in the limit of extremely large transmit power, and not applicable for any realistic transmit power level.

This problem is unique to greater than two-hop relay network and is not well understood. To avoid this situation, a rate penalty of one-third is unavoidable for both full-duplex and half-duplex relay operation, where every third relay stage is switched on alternatively one at a time. For example, in first time slot communication happens between relay stages $0--1,3--4,6--7, \ldots$, while in the second time slot communication happens between relay stages $1--2,4--5,7--8, \ldots$, in third time communication happens between relay stages $2--3,5--6,8--9, \ldots$, and so on, with periodic repetitions.

\subsection{Problem formulation}

Definition 1. (STBC) [30] A rate- $L / T T \times N_{t}$ design $\mathbf{D}$ is a $T \times N_{t}$ matrix with entries that are complex linear combinations of $L$ complex variables $s_{1}, s_{2}, \ldots, s_{L}$ and their complex conjugates. A rate- $L / T T \times N_{t} \mathrm{STBC} \mathbf{S}$ is a set of $T \times N_{t}$ matrices that are obtained by allowing the $L$ variables $s_{1}, s_{2}, \ldots, s_{L}$ of the rate- $L / T T \times N_{t}$ design $\mathbf{D}$ to take values from a finite subset $\mathbb{C}^{f}$ of the complex field $\mathbb{C}$. The cardinality of $\mathbf{S}=\left|\mathbb{C}^{f}\right|^{L}$, where $\left|\mathbb{C}^{f}\right|$ is the cardinality of $\mathcal{C}$. We refer to $s_{1}, s_{2}, \ldots, s_{L}$ as the constituent symbols of the STBC.

Definition 2. A DSTBC $\Phi$ for an $N$-hop network is a collection of STBCs $\left\{\mathbf{S}_{0}, \mathbf{S}_{1}, \ldots, \mathbf{S}_{N-1}\right\}$, where $\mathbf{S}_{0}$ is the STBC transmitted by the source and $\mathbf{S}_{n}=$ $\left[\phi_{n}^{1}\left(\mathbf{S}_{n-1}\right) \ldots \phi_{n}^{M_{n}}\left(\mathbf{S}_{n-1}\right)\right]$ is the STBC transmitted by relay stage $n$, where $\phi_{n}^{j}\left(\mathbf{S}_{n-1}\right)$ is the vector transmitted by the $j$ th relay of stage $n$ which is a function of $\mathbf{S}_{n-1}, j=$ $0, \ldots, M_{n}, n=1, \ldots, N-1$. An example of a DSTBC is illustrated in Figure 2. 

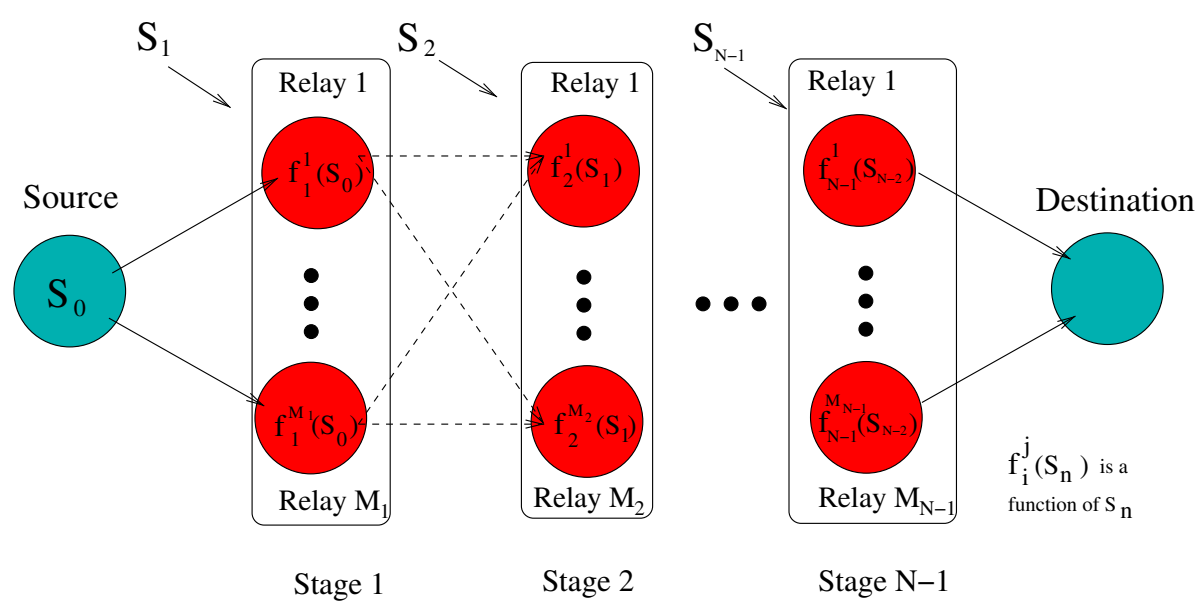

Stage N-1

Figure 2 An illustration Of the DSTBC design problem.

Definition 3. The diversity gain $[1,3]$ of a DSTBC $\Phi$ is defined as

$$
d_{\Phi}=-\lim _{E \rightarrow \infty} \frac{\log P_{e}(E)}{\log E}
$$

$P_{e}(E)$ is the pairwise error probability using DSTBC $\Phi$, and $E$ is the sum of the transmit power used by each node in the network.

The problem we consider in this article is to design DSTBCs that achieve the maximum diversity gain in an $N$-hop network. To identify the limits on the maximum possible diversity gain in an $N$-hop network, an upper bound on the diversity gain achievable with any DSTBC is presented next.

Theorem 1. The diversity gain $d_{\Phi}$ of DSTBC $\Phi$ for an $N$-hop network is upper bounded by $\min \left\{M_{n} M_{n+1}\right\}$, $n=0,1, \ldots, N-1$.

\section{Proof. See Proposition 2.1 of [23].}

Theorem 1 implies that the maximum diversity gain achievable in an $N$-hop network is equal to the minimum of the maximum diversity gain achievable between any two relay stages, when all the relays in each relay stage are allowed to collaborate. In the next section, we propose COSTBCs that are shown to achieve this upper bound on the diversity gain.

\section{COSTBC}

In this section, we introduce the COSTBC design for an $N$-hop network. Before introducing COSTBCs, we need the following definitions.
Definition 4. With $T \geq N_{t}$, a rate $L / T T \times N_{t}$ STBC $\mathbf{S}$ is called full-rank or fully diverse or is said to achieve the maximum diversity gain if the difference of any two matrices $\mathbf{M}_{1}, \mathbf{M}_{2} \in \mathbf{S}$ is full-rank, i.e.,

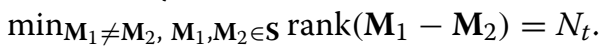

Definition 5. A rate- $L / K K \times K$ STBC $\mathbf{S}$ is called an OSTBC if the design $\mathbf{D}$ from which it is derived is orthogonal, i.e.,DD* $=\left(\left|s_{1}\right|^{2}+\cdots+\left|s_{L}\right|^{2}\right) \mathbf{I}_{K}$.

Definition 6. Let $\mathbf{S}$ be a rate- $L / K K \times K$ STBC. Then, if the maximum likelihood (ML) decoding of $\mathbf{S}$ is such that each of the constituent symbols $s_{i}, i=1, \ldots, L$ of $\mathbf{S}$ can be decoded independently of $s_{j} \forall i \neq j i, j=$ $1, \ldots, L$, then $\mathbf{S}$ is called a single symbol decodable STBC.

Remark 2. OSTBCs are single symbol decodable STBCs [25].

With these definitions we are now ready to describe COSTBCs for an $N$-hop network.

A COSTBC is a DSTBC where at each relay stage an OSTBC is transmitted, i.e., $\mathbf{S}_{n}, n=0,1, \ldots, N-1$ is an OSTBC. To construct COSTBC, the source transmits a rate- $L / M_{0} M_{0} \times M_{0}$ OSTBC $S_{0}$ in a time slot of duration $M_{0}$. The received signal $\mathbf{y}_{k}^{1} \in \mathbb{C}^{M_{0} \times 1}$ at relay $k$ of relay stage 1 can be written as

$$
\mathbf{y}_{k}^{1}=\sqrt{E_{0}} \mathbf{S}_{0} \mathbf{h}_{k}+\mathbf{z}_{k}^{1}
$$

where $\mathbb{E}\left\{\operatorname{tr}\left(\mathbf{S}_{0}^{*} \mathbf{S}_{0}\right)\right\}=M_{0}, E_{0}$ is the power transmitted by the source at each time instant, and $\mathbf{z}_{k}^{1}$ is the noise vector with i.i.d. $\mathcal{C} N(0,1)$ distributed entries. Since $\mathbf{S}_{0}$ 
is an OSTBC, using CSI, the received signal $\mathbf{y}_{k}^{1}$ can be transformed into $\tilde{\mathbf{y}}_{k}^{1} \in \mathbb{C}^{L \times 1}[1]$,

$$
\tilde{\mathbf{y}}_{k}^{1}=\sqrt{E_{0}} \underbrace{\left[\begin{array}{ccc}
\sum_{m=1}^{M_{0}}\left|h_{m k}\right|^{2} & 0 & 0 \\
0 & \ddots & 0 \\
0 & 0 & \sum_{m=1}^{M_{0}}\left|h_{m k}\right|^{2}
\end{array}\right]}_{\mathrm{H}_{k}^{1}} \mathbf{s}+\mathbf{n}_{k}^{1},
$$

where $\mathbf{s}=\left[s_{1}, s_{2}, \ldots, s_{L}\right]^{T}$ is the vector of the constituent symbols of the OSTBC $\mathbf{S}_{0}, \mathrm{H}_{k}^{1}$ is an $L \times L$ matrix, and $\mathbf{n}_{k}^{1}$ is the noise vector with i.i.d. $\mathcal{C} N\left(0, M_{0}\right)$ distributed entries. Normalizing $\tilde{y}_{k}^{1}$ by $\left(\sqrt{\mathrm{H}_{k}^{1}}\right)^{-1}$ we obtain $\hat{y}_{k}^{1}$, where

$$
\hat{\mathbf{y}}_{k}^{1}:=\left(\sqrt{\mathrm{H}_{k}^{1}}\right)^{-1} \tilde{\mathbf{y}}_{k}^{1}=\sqrt{E_{0} \mathrm{H}_{k}^{1}} \mathbf{s}+\underbrace{\left(\sqrt{\mathrm{H}_{k}^{1}}\right)^{-1} \mathbf{n}_{k}^{1}}_{\hat{\mathbf{n}}_{k}^{1}},
$$

where $\hat{\mathbf{n}}_{k}^{1}$ is an $L \times 1$ vector with entries that are uncorrelated and $\mathcal{C} N(0,1)$ distributed.

In the second time slot of duration $M_{1}$, relay $k$ of relay stage transmits $\mathbf{t}_{k}^{1}$, constructed from the signal (3)

$$
\mathbf{t}_{k}^{1}=\sqrt{\frac{E_{1} M_{1}}{L \gamma}}\left(\mathbf{A}_{k}^{1} \hat{\mathbf{y}}_{k}^{1}+\mathbf{B}_{k}^{1} \hat{\mathbf{y}}_{k}^{1 \dagger}\right)
$$

where $\gamma=\mathbb{E}\left\{\hat{\mathbf{y}}_{k}^{1 *} \hat{\mathbf{y}}_{k}^{1}\right\}$ to ensure that the average power transmitted by each relay at any time instant is $E_{1}$, i.e., $\mathbb{E}\left\{\mathbf{t}_{k}^{1 \dagger} \mathbf{t}_{k}^{1}\right\}=E_{1}, \mathbf{A}_{k}^{1}, \mathbf{B}_{k}^{1}$ are $M_{1} \times L$ matrices such that

$$
\begin{aligned}
\mathbf{A}_{k}^{1 *} \mathbf{B}_{k}^{1} & =-\mathbf{B}_{k}^{1 *} \mathbf{A}_{k}^{1}, \text { and } \operatorname{tr}\left(\mathbf{A}_{k}^{1 *}(l) \mathbf{A}_{k}^{1}(l)+\mathbf{B}_{k}^{1 *}(l) \mathbf{B}_{k}^{1}(l)\right) \\
& =1,
\end{aligned}
$$

$\forall k=1,2 \ldots, M_{1}, \quad l=1,2, \ldots L$, where $\mathbf{A}_{k}^{1}(l)$ and $\mathbf{B}_{k}^{1}(l)$ denote the $l$ th column of $\mathbf{A}_{k}$ and $\mathbf{B}_{k}$, respectively, and $\mathbf{S}_{1}:=\left[\mathbf{A}_{1}^{1} \mathbf{s}+\mathbf{B}_{1}^{1} \mathbf{s}^{\dagger} \ldots \mathbf{A}_{M_{1}}^{1} \mathbf{s}+\mathbf{B}_{M_{1}}^{1} \mathbf{s}^{\dagger}\right]$ is an OSTBC.

Under these assumptions, the $M_{1} \times 1$ received signal at the $i^{\text {th }}$ relay of relay stage 2 is for $i=1,2, \ldots M_{2}$, where $\mathbf{f}_{i}^{1}=\left[f_{1 i}^{1} \ldots f_{M_{1} i}^{1}\right]^{T}, \mathbf{z}_{i}^{2}$ is the noise vector with i.i.d. $\mathcal{C} N(0,1)$ entries and

$\hat{\mathbf{H}}^{\frac{1}{2}}=\left[\begin{array}{cccc}\sqrt{\sum_{m=1}^{M_{0}}\left|h_{m 1}\right|^{2}} & 0 & 0 & 0 \\ 0 & \sqrt{\sum_{m=1}^{M_{0}}\left|h_{m 2}\right|^{2}} & 0 & 0 \\ 0 & 0 & \ddots & 0 \\ 0 & 0 & 0 & \sqrt{\sum_{m=1}^{M_{0}}\left|h_{m M_{1}}\right|^{2}}\end{array}\right]$.

Note that

$$
\mathbf{y}_{i}^{2}=\sqrt{\frac{E_{0} E_{1} M_{1}}{L \gamma}} \mathbf{S}_{1} \hat{\mathbf{H}}^{\frac{1}{2}} \mathbf{f}_{i}^{1}+\mathbf{w}_{i}^{2}
$$

is of the form (1), where $\boldsymbol{S}_{1}$ is also an OSTBC similar to $\mathbf{S}_{0}$. Thus, repeating the operations illustrated in (2), (3), and (4), and using matrices $\mathbf{A}_{k}^{n}, \mathbf{B}_{k}^{n}, k=1, \ldots, M_{n}, n=$ $2, \ldots, N-1$ satisfying (5), an OSTBC is transmitted from each relay stage to construct the COSTBC.

Using COSTBCs, let the received signal at the $k$ th antenna of the destination be

$$
\mathbf{y}_{k}^{N}=\sqrt{\theta E_{N-1}} \mathbf{S}_{N-1} \mathbf{c}_{k}+\mathbf{w}_{k}^{N}
$$

where $\theta$ is such that the average power transmitted from the $N-1$ th relay stage is $E_{N-1}, \mathbf{c}_{k} \in \mathbb{C}^{M_{N-1} \times 1}$ is the equivalent channel vector between the source and the $k$ th antenna of the destination, and $\mathbf{w}_{k}^{N}$ is the noise vector. Let $\mathbf{y}^{N}:=\left[\left(\mathbf{y}_{1}^{N}\right)^{T} \ldots\left(\mathbf{y}_{M_{N}}^{N}\right)^{T}\right]^{T}, \mathbf{c}:=\left[\mathbf{c}_{1}^{T} \ldots \mathbf{c}_{M_{N}}^{T}\right]^{T}$, and $\mathbf{w}:=\left[\left(\mathbf{w}_{1}^{N}\right)^{T} \ldots\left(\mathbf{w}_{M_{N}}^{N}\right)^{T}\right]^{T}$, then with the ML decoding rule, using the received signal from all antennas $\mathbf{y}^{N}, \mathbf{S}^{\star}$ is decoded if

$$
\begin{aligned}
& \mathbf{S}^{\star}=\arg \min _{\mathbf{S}_{N-1}}\left\|\mathbf{R}^{-1 / 2} \mathbf{y}^{N}-\mathbf{R}^{-1 / 2} \mathbf{S}_{N-1} \mathbf{c}\right\|^{2}, \\
& =\arg \min _{\mathbf{S}_{N-1}} \mathbf{c}^{*} \mathbf{S}_{N-1}^{*} \mathbf{R}^{-1} \mathbf{y}^{N}-\mathbf{c}^{*} \mathbf{S}_{N-1}^{*} \mathbf{R}^{-1} \mathbf{S}_{N-1} \mathbf{c},
\end{aligned}
$$

$$
\begin{aligned}
& \mathbf{y}_{i}^{2}=\sum_{k=1}^{M_{1}} \mathbf{t}_{k}^{1} f_{k i}^{1}+\mathbf{z}_{i}^{2} \\
& =\sqrt{\frac{E_{0} E_{1} M_{1}}{L \gamma}} \underbrace{\left[\mathbf{A}_{1}^{1} \mathbf{s}+\mathbf{B}_{1}^{1} \mathbf{s}^{\dagger} \mathbf{A}_{2}^{1} \mathbf{s}+\mathbf{B}_{2}^{1} \mathbf{s}^{\dagger} \ldots \mathbf{A}_{M_{1}}^{1} \mathbf{s}+\mathbf{B}_{M_{1}}^{1} \mathbf{s}^{\dagger}\right]}_{\mathbf{S}_{1}} \hat{\mathbf{H}}^{\frac{1}{2}} \mathbf{f}_{i}^{1} \\
& +\underbrace{\sqrt{\frac{E_{1} M_{1}}{L \gamma}}\left[\mathbf{A}_{1}^{1} \hat{\mathbf{n}}_{1}^{1}+\mathbf{B}_{1}^{1} \hat{\mathbf{n}}_{1}^{1 \dagger} \ldots \mathbf{A}_{M_{1}}^{1} \hat{\mathbf{n}}_{M_{1}}^{1}+\mathbf{B}_{M_{1}}^{1} \hat{\mathbf{n}}_{M_{1}}^{1 \dagger}\right] \mathbf{f}_{i}^{1}+\mathbf{z}_{i}^{2}}_{\mathbf{w}_{i}^{2}},
\end{aligned}
$$


where $\mathbf{R}:=\mathbb{E}\left\{\mathbf{w} \mathbf{w}^{*}\right\}$ is the noise covariance matrix. Note that if $\mathbf{R}$ is a scaled identity matrix, then the ML decision rule (8) is equal to $\sum_{j=1}^{L} \min _{j} f\left(s_{j}\right)$, where $f\left(s_{j}\right)$ is a function of $s_{j}$ that does not depend on $s_{k}, k \neq j$, since $\mathbf{S}_{N-1}^{*} \mathbf{S}_{N-1}$ is a scaled identity matrix. Thus, the COSTBCs are single symbol decodable if $\mathbf{R}$ is a scaled identity matrix.

With COSTBC, for $j \geq 2$, the noise vectors $\mathbf{w}_{i}^{j}$ received at the $i$ th antenna of relay stage $j$ are correlated for $i=$ $1, \ldots, M_{j}$, since all the noise components of the signals transmitted from the previous relay stages $\hat{\mathbf{n}}_{1}^{j-1} \ldots \hat{\mathbf{n}}_{M_{j-1}}^{j-1}$ have a contribution in all the $\mathbf{w}_{i}^{j}, i=1, \ldots, M_{j}$. For example in (6), $\mathbf{w}_{i}^{2}$ has contribution from $\hat{\mathbf{n}}_{k}^{1}$ (3) $\forall k=$ $1, \ldots M_{1}$. Thus, in general, the noise covariance matrix $\mathbf{R}$ with COSTBC is not a scaled identity matrix (not even diagonal), and hence the COSTBCs are not single symbol decodable. For a special case of $N=2$, and $M_{2}=1$, the noise covariance matrix $\mathbf{R}$ is diagonal, however, not a scaled identity matrix. Moreover, with $N=2$, and $M_{2}=1$, an interesting property of COSTBC is that by adding some channel coefficient-dependent noise terms to the received signal, the noise covariance matrix $\mathbf{R}$ can be made a scaled identity matrix as described in Appendix 2. Thus, compared to the ML detection of COSTBCs that entails joint decoding of symbols, by degrading the received signal and paying a penalty in terms of coding gain, COSTBCs are single symbol decodable and hence have linear decoding complexity. A performance comparison with added noise term is illustrated in Figure 3.
Even though COSTBCs are not single symbol decodable, we next show that with COSTBCs, maximum diversity gain can be achieved if the destination decodes all constituent symbols of COSTBCs independently of each other according to rule (10). Exploiting the orthogonality property of the OSTBCs transmitted by each relay stage, the received signal at the destination can be separated in terms of individual symbols transmitted by the source as follows. From (7), since $\mathbf{S}_{N-1}$ is an OSTBC, similar to (2), the received signal at the $k$ th antenna of the destination can be transformed as

$$
\tilde{\mathbf{y}}_{k}^{N}=\sqrt{\theta E_{N-1}} \underbrace{\left[\begin{array}{ccc}
\left|\mathbf{c}_{k}\right|^{2} & 0 & 0 \\
0 & \ddots & 0 \\
0 & 0 & \left|\mathbf{c}_{k}\right|^{2}
\end{array}\right]} \mathbf{s}+\mathbf{z}_{k}^{N}
$$

where $\mathbf{s}=\left[s_{1}, s_{2}, \ldots, s_{L}\right]^{T}$ is the vector of the constituent symbols of the OSTBC $\mathbf{S}_{0}$, and $\mathbf{z}_{k}^{N}$ is the correlated noise vector. Combining the transformed signals from all $M_{N}$ antennas, the received signal can be written in terms of individual constituent symbols of the OSTBC $\mathbf{S}_{0}$, as

$$
y_{\ell}^{N}=\sum_{k=1}^{M_{n}} c_{k} s_{\ell}+z_{\ell},
$$

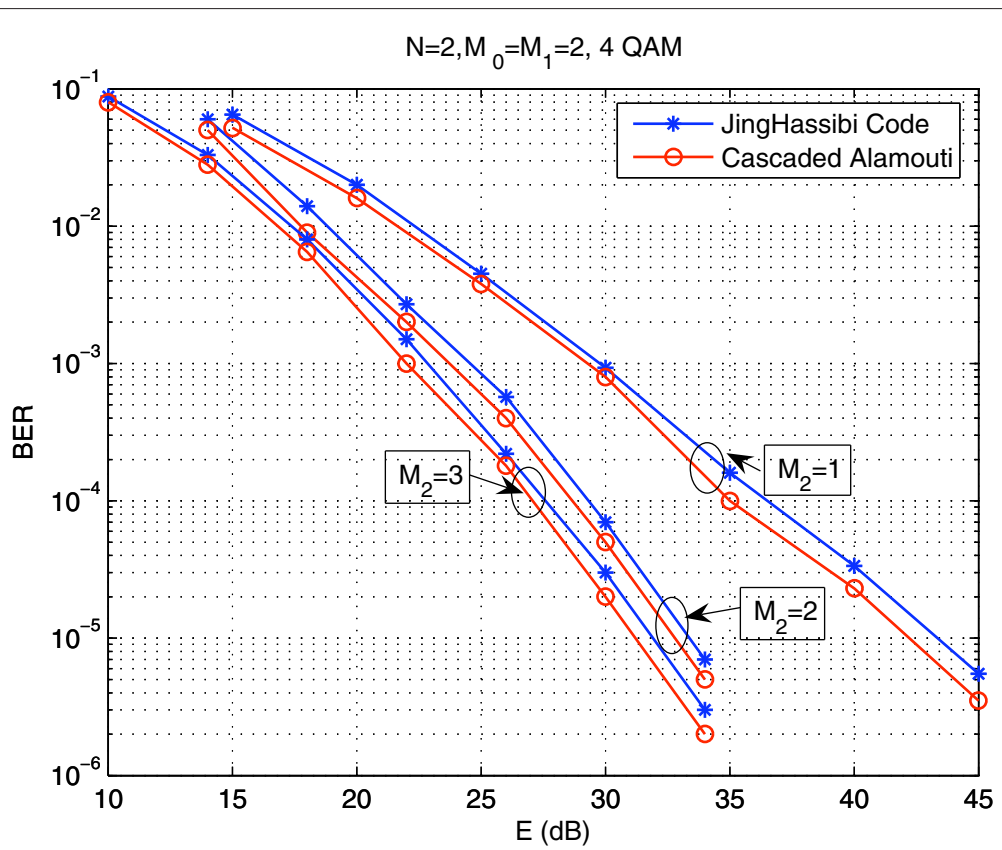

Figure 3 BER cascaded Alamouti code with ML, independent decoding, ML with added noise, code for $N=2$-hop network. 
where $c_{i}=\left|\mathbf{c}_{i}\right|^{2}$ and $z_{\ell}=\sum_{k=1}^{M_{n}} \mathbf{z}_{k}^{N}(\ell)$ for $\ell=1,2, \ldots, L$. Thus, each symbol $s_{\ell}$ can be decoded independently using the rule

$$
s_{\ell}=\arg \min _{s}\left\|y_{\ell}^{N}-\sum_{k=1}^{M_{n}} c_{k} s\right\|^{2}
$$

even though this is not ML decoding. We consider this decoding rule to ensure linear decoding complexity and show that COSTBCs achieve maximum diversity gain with this rule.

Theorem 2. COSTBCs achieve the diversity gain upper bound(Theorem 1) in an $N$-hop network with decoding rule (10).

\section{Proof. See Appendix 1.}

The basic idea behind the proof of Theorem 2 is to exploit the orthogonality of OSTBCs transmitted by each relay stage.

For the special case of $N=2$ and $M_{2}=1$, we next show that the COSTBCs can be made single symbol decodable by degrading the received signal by adding some channel coefficient-dependent noise terms as discussed before.

Theorem 3. COSTBCs are single symbol decodable STBCs after adding some channel coefficient-dependent noise terms to the received signals for $N=2$ and $M_{2}=1$.

\section{Proof. See Appendix 2.}

Remark 3. CSI: We note that for decoding of COSTBCs, global CSI is required at the destination. The requirement of destination having global CSI regarding all the underlying channels has been made in several recent related papers, including [23,24]. Actually, this is a common assumption made by all papers that consider amplify-andforward protocol. Since mostly, only two-hop communication is considered, the CSI requirement is somewhat limited compared to the case of multi-hop communication, the topic of this paper and [22-24]. Acquiring such CSI in practice is a challenge, however, using techniques like Grassmannian codebooks, CSI about all channels can be acquired by the destination through the relay nodes by dedicating the start of time slots for training purposes. In particular, relay in stage 1 can get the CSI between source and itself by using pilots and channel estimation. Thereafter, by using Grassmannian codebooks it can forward the CSI it has gathered to the next relay stage in addition to sending pilots for the relays in the next stage to gather CSI between relay stages. Repeating this procedure all nodes can get the required CSI.
Another assumption about CSI we make for our code construction to work is that CSI is available at each relay node for channels preceding itself which is not required for other related works [22-24]. Since the CSI required at the destination for any amplify-and-forward protocol has to be transmitted through to the destination through the relays, CSI can safely be assumed to be available at each relay node as well. Thus, this is also not a limiting assumption.

Discussion: In this section, we constructed COSTBCs by cascading OSTBCs at each relay stage. OSTBCs are cascaded at each relay stage by first separating each constellation symbol of the OSTBC transmitted from the preceding relay stage, and then transmitting another OSTBC to the next relay stage. The proposed OSTBC cascading strategy is novel, and different than other approaches that use Alamouti code or OSTBC in a distributed manner [12,31].

We showed that the single symbol decodable property of OSTBCs is lost by cascading OSTBCs to construct COSTBCs. Using the orthogonality property of the OST$\mathrm{BCs}$, however, we showed that the maximum diversity gain can be achieved by COSTBCs even when each source transmitted symbol is decoded independently. Therefore, COSTBCs have decoding complexity that is linear in the number of symbols transmitted by source in one codeword, which is quite critical for practical implementations. Since independent symbol decoding is not ML, COSTBCs entail an unavoidable coding gain loss, however, we show that at least in terms of diversity gain there is no loss compared to ML decoding. We also showed that the COSTBCs are single symbol decodable for a two-hop wireless network $N=2$ when the destination has only a single antenna $M_{2}=1$, by adding some channel coefficient-dependent noise terms to the received signal.

\section{Explicit code constructions}

In this section, we explicitly construct COSTBCs that achieve the maximum diversity gain in an $N$-hop network. The ingredient OSTBCs can be borrowed from $[25,32,33]$, similar to [34]. We present examples of COSTBCs for $N=2, M_{0}=M_{1}=2$ using the Alamouti code [26], $N=2, \quad M_{0}=M_{1}=4$ using the rate- $3 / 4$ 4 antenna OSTBC [25] and $N=2, \quad M_{0}=M_{1}=4$ using the rate-3/4 4 antenna OSTBC and the Alamouti code.

Example 1. (Cascaded Alamouti code) We consider $N=2, M_{0}=M_{1}=2$ case and let $\mathbf{S}_{0}$ be the Alamouti code given by $\mathbf{S}_{a l a}=\left[\begin{array}{cc}s_{1} & s_{2} \\ -s_{2}^{*} & s_{1}^{*}\end{array}\right]$ where $s_{1}$ and $s_{2}$ are 
constituent symbols of the Alamouti code. The $2 \times 1$ received signal at relay $m$ is

$$
\left[\begin{array}{l}
y_{1 m} \\
y_{2 m}
\end{array}\right]=\sqrt{E_{0}}\left[\begin{array}{cc}
s_{1} & s_{2} \\
-s_{2}^{*} & s_{1}^{*}
\end{array}\right]\left[\begin{array}{l}
h_{1 m} \\
h_{2 m}
\end{array}\right]+\left[\begin{array}{l}
n_{1 m} \\
n_{2} m
\end{array}\right]
$$

for $m=1,2$. Transforming this in the usual way

$$
\left[\begin{array}{c}
y_{1 m} \\
-y_{2 m}^{*}
\end{array}\right]=\sqrt{E_{0}} \underbrace{\left[\begin{array}{cc}
h_{1 m} & h_{2 m} \\
-h_{2 m}^{*} & h_{1 m}^{*}
\end{array}\right]}_{\tilde{\mathbf{H}}_{m}}\left[\begin{array}{l}
s_{1} \\
s_{2}
\end{array}\right]+\left[\begin{array}{c}
n_{1 m} \\
-n_{2 m}^{*}
\end{array}\right]
$$

for $m=1,2$. We define $\tilde{h}_{m}:=\left|h_{1 m}\right|^{2}+\left|h_{2 m}\right|^{2}, \eta_{1 m}:=$ $\left(n_{1 m} h_{1 m}^{*}+n_{2 m}^{*} h_{2 m}\right)$, and $\eta_{2 m}:=\left(n_{1 m} h_{2 m}^{*}-n_{2 m}^{*} h_{1 m}\right)$.

Pre-multiplying by $\tilde{\mathbf{H}}_{m}^{*}$,

$$
\begin{aligned}
{\left[\begin{array}{ll}
\hat{y}_{1 m} & \hat{y}_{2 m}^{*}
\end{array}\right]^{T}: } & =\tilde{\mathbf{H}}_{m}^{*}\left[\begin{array}{ll}
y_{1 m} & -y_{2 m}^{*}
\end{array}\right]^{T} \\
& =\sqrt{E_{0}}\left[\begin{array}{ll}
\tilde{h}_{m} s_{1} & \tilde{h}_{m} s_{2}
\end{array}\right]^{T}+\left[\begin{array}{ll}
\eta_{1 m} & \eta_{2 m}
\end{array}\right]^{T}
\end{aligned}
$$

for $m=1,2$.

Now using

$$
\mathbf{A}_{1}=\left[\begin{array}{ll}
1 & 0 \\
0 & 1
\end{array}\right], \quad \mathbf{B}_{1}=\mathbf{0}_{2}, \quad \mathbf{A}_{2}=\mathbf{0}_{2}, \mathbf{B}_{2}=\left[\begin{array}{cc}
0 & -1 \\
1 & 0
\end{array}\right]
$$

the STBC $\mathbf{S}_{1}$ formed by the two relays is equal to $\mathbf{S}_{\text {ala }}^{T}$ which is also an OSTBC as required. Note that $\mathbf{A}_{i}, \mathbf{B}_{i} i=$ 1,2 satisfy the requirements of (5). We call this the cascaded Alamouti code.

Example 2. In this example, we consider the case $N=2$, $M_{0}=4, M_{1}=4$. We choose $\mathbf{S}_{0}$ to be the rate-3/4 OSTBC for 4 transmit antennas given by

$$
\mathbf{S}_{0}=\left[\begin{array}{cccc}
s_{1} & s_{2} & s_{3} & 0 \\
-s_{2}^{*} & s_{1}^{*} & 0 & s_{3} \\
s_{3}^{*} & 0 & -s_{1}^{*} & s_{2} \\
0 & s_{3}^{*} & -s_{2}^{*} & -s_{1}
\end{array}\right]
$$

and use

$$
\begin{aligned}
& \mathbf{A}_{1}=\left[\begin{array}{lll}
1 & 0 & 0 \\
0 & 0 & 0 \\
0 & 0 & 0 \\
0 & 0 & 0
\end{array}\right], \mathbf{A}_{2}=\left[\begin{array}{lll}
0 & 1 & 0 \\
0 & 0 & 0 \\
0 & 0 & 0 \\
0 & 0 & 0
\end{array}\right], \\
& \mathbf{A}_{3}=\left[\begin{array}{lll}
0 & 0 & 1 \\
0 & 0 & 0 \\
0 & 0 & 0 \\
0 & 0 & 0
\end{array}\right], \mathbf{A}_{4}=\left[\begin{array}{lll}
0 & 0 & 0 \\
0 & 0 & 1 \\
0 & 1 & 0 \\
-1 & 0 & 0
\end{array}\right]
\end{aligned}
$$

and

$$
\begin{aligned}
\mathbf{B}_{1}=\left[\begin{array}{ccc}
0 & 0 & 0 \\
0 & -1 & 0 \\
0 & 0 & 1 \\
0 & 0 & 0
\end{array}\right], \mathbf{B}_{2}=\left[\begin{array}{lll}
0 & 0 & 0 \\
1 & 0 & 0 \\
0 & 0 & 0 \\
0 & 0 & 1
\end{array}\right], \\
\mathbf{B}_{3}=\left[\begin{array}{ccc}
0 & 0 & 0 \\
0 & 0 & 0 \\
-1 & 0 & 0 \\
0 & -1 & 0
\end{array}\right], \mathbf{B}_{4}=\left[\begin{array}{lll}
0 & 0 & 0 \\
0 & 0 & 0 \\
0 & 0 & 0 \\
0 & 0 & 0
\end{array}\right] .
\end{aligned}
$$

It is easy to verify that $\operatorname{tr}\left(\mathbf{A}_{i}^{*} \mathbf{A}_{i}+\mathbf{B}_{i}^{*} \mathbf{B}_{i}\right)=3$ and $\mathbf{A}_{i}^{*} \mathbf{B}_{i}=-\mathbf{B}_{i}^{*} \mathbf{A}_{i}, \quad i=1,2,3,4$ as required. Then the STBC $\mathbf{S}_{1}=\mathbf{S}_{0}$ using these $\mathbf{A}_{i}, \mathbf{B}_{i} i=1,2,3,4$, which is a rate-3/4 OSTBC as described above.

In both the previous examples, we constructed a COSTBC for the $N=2$-hop case by repeatedly using the same OSTBC at both the source and the relay stage. Using a similar procedure, it can be seen that when $M_{i}=$ $M_{j} \forall i, j=0,1, \ldots, N-1, i \neq j$ we can construct COSTBCs by using particular OSTBC for $M_{0}$ antennas at the source and each relay stage, e.g., if $\mathcal{O}$ is an OSTBC for $M_{0}$ antennas, then by using $\mathbf{S}_{n}=\mathcal{O}, n=0,1, \ldots, N-1$ we obtain a maximum diversity gain achieving COSTBC. OSTBC constructions for different number of antennas can be found in [25]. In the next example, we construct COSTBC for $M_{0}=4$ and $M_{1}=2$ by cascading the rate-3/4 4 antenna OSTBC with the Alamouti code.

Example 3. Let $N=2, M_{0}=4$, and $M_{1}=2$. We choose $S_{0}$ to be the rate-3/4 4 antenna OSTBC (12) and $\mathbf{S}_{1}$ to be the Alamouti code. The COSTBC is constructed as follows.

Let $\mathbf{S}_{0}$ given by (12) be the transmitted rate$3 / 44$ antenna OSTBC from the source. Then the received signal at relay node $m, m=1,2$ is

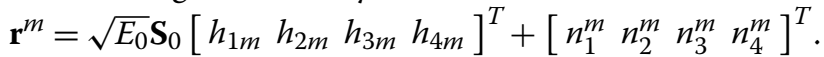
Using CSI the received signal $\mathbf{r}^{m}$ can be transformed into $\hat{\mathbf{r}}^{m}$, where $\hat{\mathbf{r}}^{m}:=\left[\begin{array}{lll}\hat{r}_{1}^{m} & \hat{r}_{2}^{m} & \hat{r}_{3}^{m}\end{array}\right]^{T}=$ $\sqrt{E_{0}}\left[\begin{array}{lll}\hat{h}_{m} s_{1} & \hat{h}_{m} s_{2} & \hat{h}_{m} s_{3}\end{array}\right]^{T}+\left[\begin{array}{lll}\hat{n}_{1}^{m} & \hat{n}_{2}^{m} & \hat{n}_{3}^{m}\end{array}\right]^{T}$ and $\hat{h}_{m}=$ $\sqrt{\sum_{i=1}^{M_{0}}\left|h_{i m}\right|^{2}}$. Then in the next time slot, the relay $m, m=1,2$ transmits $\mathbf{A}_{m}\left[\begin{array}{ll}\hat{r}_{1}^{m} & \hat{r}_{2}^{m}\end{array}\right]^{T}+\mathbf{B}_{m}\left[\begin{array}{ll}\hat{r}_{1}^{m} & \hat{r}_{2}^{m}\end{array}\right]^{T \dagger}$ where $\mathbf{A}_{m}, \mathbf{B}_{m}$ are given in (11).

These operations are repeated at the source and each relay stage in subsequent time slots. In the next time slot, signal $s_{3}$ received in the previous time slot and $s_{1}$ received in the current time slot is transmitted from relay stage 1 to the destination. Clearly, the relay stage transmits an Alamouti code which is an OSTBC and hence leads to a COSTBC construction for $M_{0}=4, M_{1}=2$.

Using a similar technique as illustrated in this example, COSTBCs can be constructed for different number of 
source antenna and relay node configurations by suitably adapting different OSTBCs.

\section{Simulation results}

In this section, we provide simulation results to illustrate the bit error rates (BERs) of COSTBCs for 2- and 3-hop networks. In all the simulation plots, $E$ denotes the total power used by all nodes in the network, i.e., $E_{0}+\sum_{n=1}^{N-1} M_{n} E_{n}=E$ and the additive noise at each relay and the destination is complex Gaussian with zero mean and unit variance. By equal power allocation between the source and each relay stage we mean $E_{0}=M_{n} E_{n}=$ $\frac{E}{N}, \forall n=1, \ldots, N-1$.

In Figure 4, we plot the BERs of a cascaded Alamouti code and the comparable DSTBC from [3] with 4 QAM modulation for $N=2, M_{0}=M_{1}=2$, and $M_{2}=1,2,3$ with equal power allocation between the source and all the relays. It is easy to see that both the cascaded Alamouti code and the DSTBC from [3] achieves the maximum diversity gain of the 2-hop network, however, COSTBCs require $1 \mathrm{~dB}$ less power than the DSTBCs from [3], to achieve the same BER. The improved BER performance of COSTBCs over DSTBCs from [3] is due to fact that with COSTBCs, each relay coherently combines the signal received from the previous relay stage before forwarding it to the next relay stage, while no such combining is done in [3]. Note that, however, DSTBCs from [3] do not need CSI at any relay, in contrast to COSTBCs which use CSI for transforming the signal and transmitting an OSTBC. To illustrate the loss with independent decoding (10) with respect to ML decoding, for $N=2$ and $M_{2}=1$, in Figure 3, we plot the BER performance of cascaded Alamouti codes with ML decoding, with independent decoding (10), and adding channel coefficient-dependent noise for which the COSTBC is single symbol decodable. We observe that even though there is a sufficient gap between ML and non-ML decodings, there is a negligible difference between independent decoding (10) and ML single symbol decoding with added noise.

We also compare the BER performance of COSTBC with perfect and imperfect CSI in Figure 5 for a 2-hop network with number of destination antennas $M_{2}=1,2$. Each relay uses channel estimation with the help of pilots to gather the necessary CSI from the source to itself. Then each relay uses a 16-bit Grassmannian codebooks [35] for relaying the CSI between source and relay it has estimated, in addition to sending pilots for the destination to get the CSI between each relay and destination. We notice that even though there is a performance loss with imperfect CSI, however, its not too significant.

Next, we plot the BER curves for $N=2, M_{0}=M_{1}=4$, and $N=2, M_{0}=4, M_{1}=2$ configurations in Figures 6 and 7 with different $M_{2}$ and using equal power allocation between the source and the relay stage. For the $M_{0}=$ $M_{1}=4$ case, we use the cascaded rate-3/4 4 antenna OSTBC and for the $M_{0}=4, M_{1}=2$ case we use a rate$3 / 44$ antenna OSTBC at the source and the Alamouti code across both the relays as discussed in Section 4. From Figures 6 and 7, it is clear that both these codes achieve

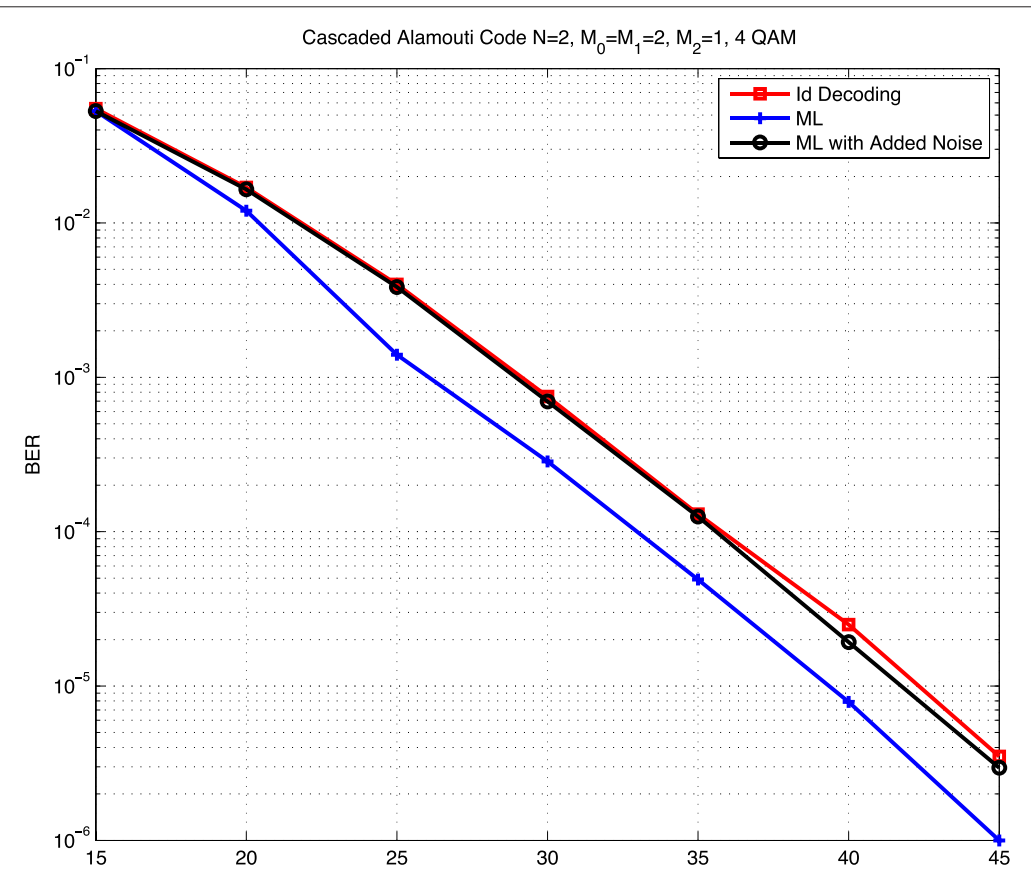

Figure 4 BER comparison of cascaded Alamouti code with JingHassibi code for $N=2$-hop network. 


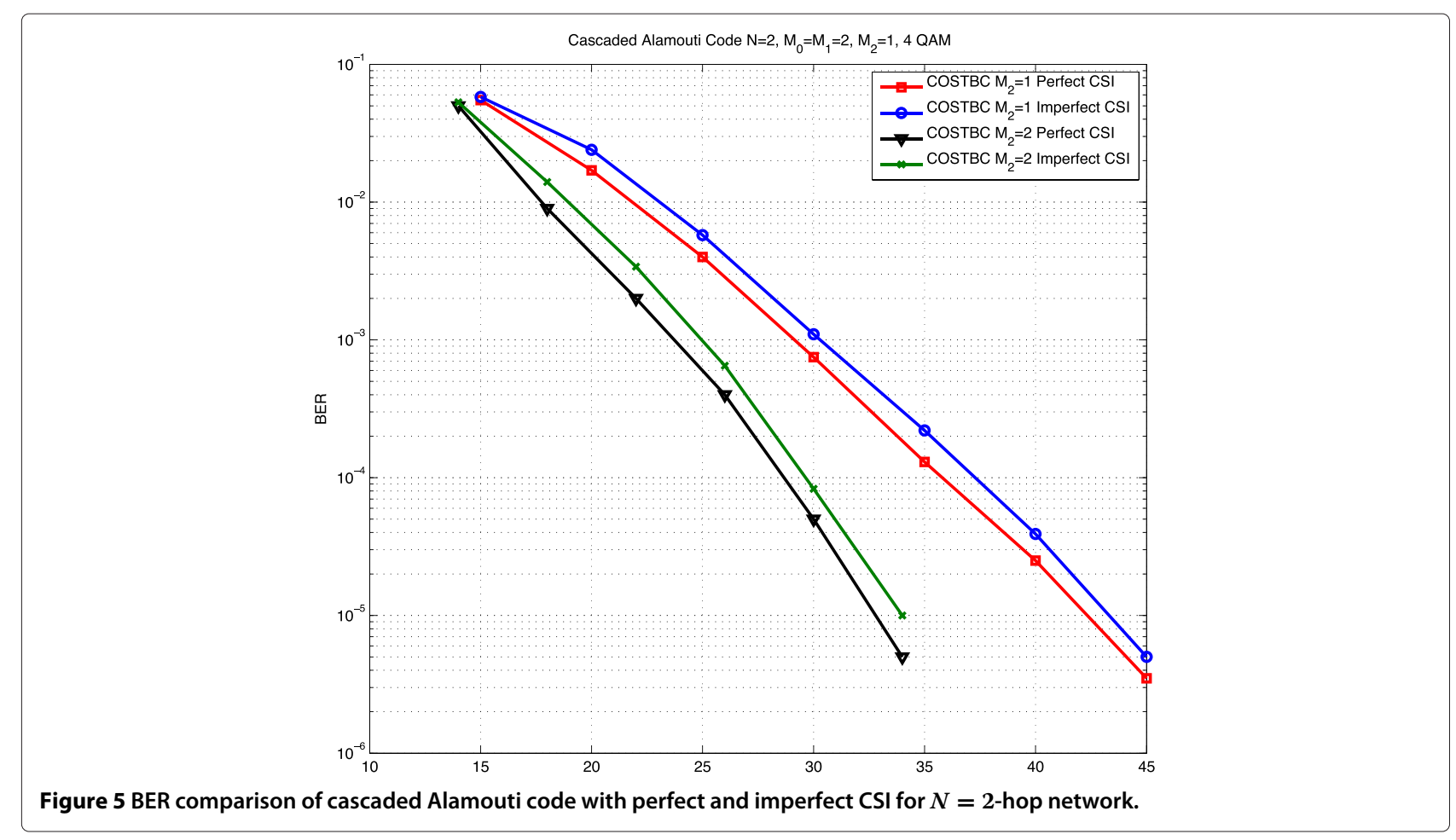

the maximum diversity gain for the respective network configurations.

Finally, in Figure 8, we plot the BERs of a cascaded Alamouti code with $N=3$-hop network where $M_{0}=$ $M_{1}=M_{2}=2$ with $M_{3}=1,2,3$, and the cascaded Alamouti code is generated by repeated use of the Alamouti code by each relay stage with equal power allocation between the source and the relay stages. In this case also, it is clear that the cascaded Alamouti code achieves the maximum diversity gain but there is an SNR loss compared to $N=2$ case, because of the noise added by one extra relay stage.

From all the simulation plots, it is clear that COST$\mathrm{BCs}$ require large transmit power to obtain reasonable

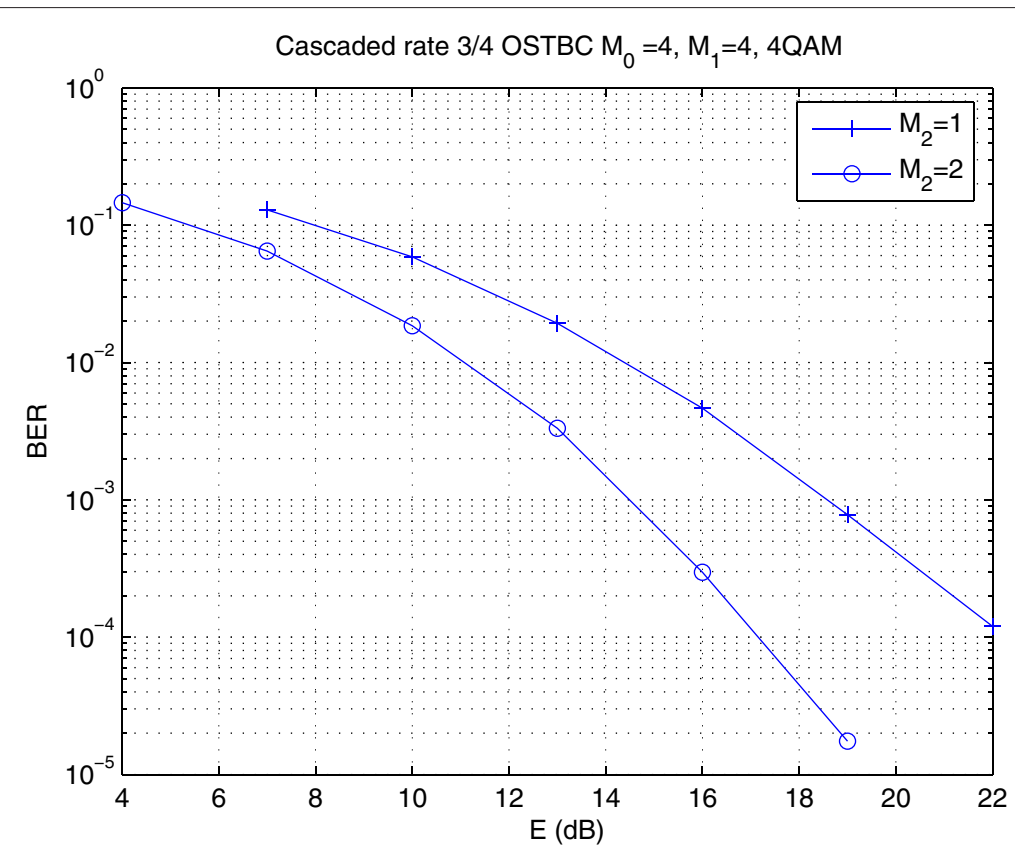

Figure 6 Cascaded rate $3 / 44$ antenna OSTBC for $M_{0}=M_{1}=4$. 


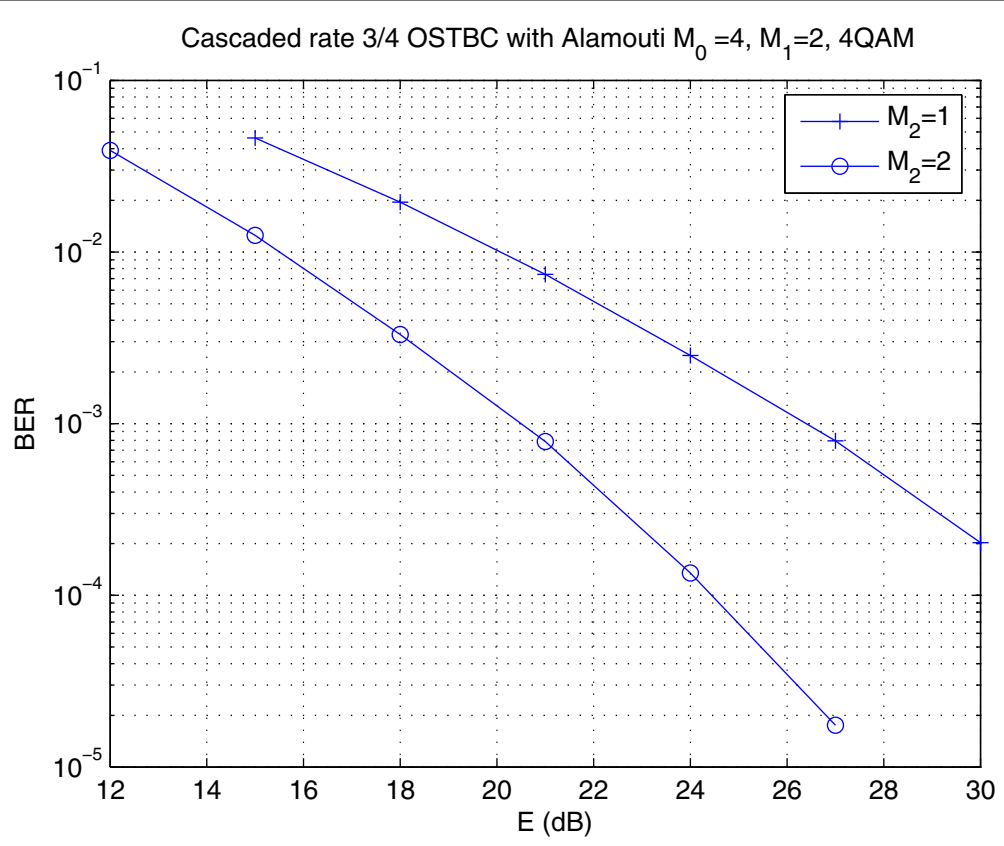

Figure 7 Cascaded rate 3/4 4 antenna OSTBC with Alamouti Code for $M_{0}=M_{1}=4$.

BERs with multi-hop wireless networks. This is a common phenomenon across all the maximum diversity gain achieving DSTBCs for multi-hop wireless networks that use AF $[3,5,9]$. With AF, the noise received at each relay gets forwarded towards the destination and limits the received SNR at the destination, however, without using AF it is difficult to achieve the maximum diversity gain in a multi-hop wireless network.

\section{Conclusion}

In this article, we designed COSTBCs that achieve the maximum diversity gain in a multi-hop wireless network with low decoding complexity. We then gave an explicit construction of COSTBCs for various numbers of source, destination, and relay antennas. The only restriction that COSTBCs impose is that the source and all the relay stages have to use an OSTBC. It is well known that high rate

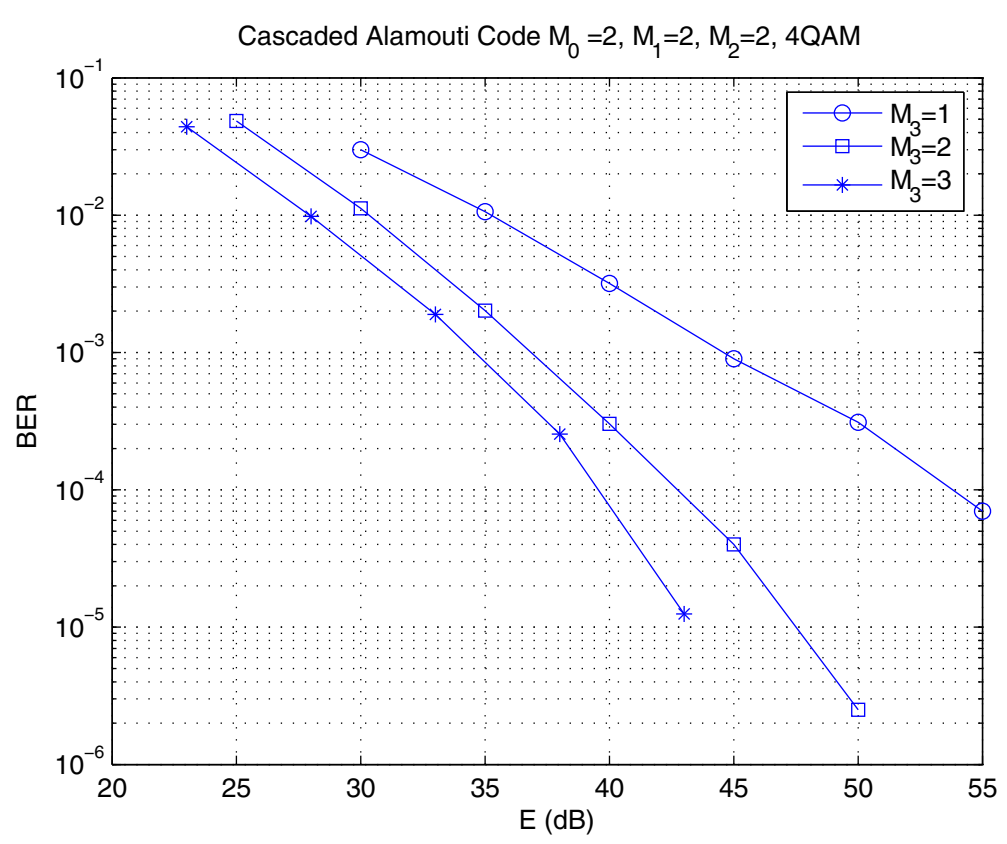

Figure 8 Cascaded Alamouti Code for $N=3$-hop network. 
OSTBCs do not exist; therefore, the COSTBCs have rate limitations. For future work it will be interesting to see whether the OSTBC requirement can be relaxed without sacrificing the maximum diversity gain and minimum decoding complexity of the COSTBCs.

\section{Appendix 1}

We prove Theorem 2 using induction. First we show that COSTBCs achieve the maximum diversity gain for $N=2$, and then extend the result for a $k$-hop network, where $k$ is any arbitrary natural number.

The outage probability $P_{\text {out }}(R)$ is defined as $P_{\text {out }}(R):=$ $P(I(\mathbf{s} ; \mathbf{r}) \leq R)$, where $\mathbf{s}$ is the input and $\mathbf{r}$ is the output of the channel and $I(\mathbf{s} ; \mathbf{r})$ is the mutual information between $\mathbf{s}$ and $\mathbf{r}$ [36]. Let $d_{\text {out }}(r)$ be the SNR exponent of $P_{\text {out }}$ with rate of transmission $R$ scaling as $r \log \mathrm{SNR}$, i.e., $\log P_{\text {out }}(r \log \mathrm{SNR}) \doteq \mathrm{SNR}^{-d_{\text {out }}(r)}$. Then, if $P_{e}(\mathrm{SNR}) \doteq$ $\mathrm{SNR}^{-d(r)}$, then from [29], and the compound channel argument [24], $P_{\text {out }}(r \log \mathrm{SNR}) \doteq P_{e}(\mathrm{SNR}), d(r) \doteq d_{\text {out }}(r)$. Therefore, to compute $d(r)$, it is sufficient to compute $d_{\text {out }}(r)$. In the following, we compute $d_{\text {out }}(r)$ for the COSTBC with a 2-hop network.

For the 2-hop network, similar to (2), from (19), the received signal at any receive antenna of the destination can be separated in terms of the individual constituent symbols of the OSTBC transmitted by the source. Similar to (2), from (19), separating the constituent symbols of $\mathbf{S}_{0}$, let $y_{\ell}^{j}$ be the received signal at the $j$ th antenna of the destination corresponding to $s_{\ell}$, the $\ell$ th, $l=1,2, \ldots, L$ symbol transmitted from the source. Then $y_{\ell}^{j}$ can be written as

$$
y_{\ell}^{j}=\sqrt{\theta E_{1}} \sum_{k=1}^{M_{1}}\left|g_{k j}\right|^{2}\left(\sum_{m=1}^{M_{0}}\left|h_{m k}\right|^{2}\right) s_{\ell}+z_{\ell}^{j}
$$

where $\theta$ is the normalization constant so as to ensure the power constraint of $E_{1}$ for the relay stage 1 , and $z_{\ell}^{j}$ is the additive white Gaussian noise (AWGN) with zero mean and $\sigma_{j}^{2}$ variance. Note that $z_{\ell}^{j}$ 's are not independent for $j=1, \ldots, M_{2}$. Adding the received signal corresponding to $s_{\ell}$ from all the antennas of the destination of the 2-hop network, we get

$$
y_{\ell}=\sqrt{\theta E} \sum_{j=1}^{M_{2}} \sum_{k=1}^{M_{1}}\left|g_{k j}\right|^{2}\left(\sum_{m=1}^{M_{0}}\left|h_{m k}\right|^{2}\right) s_{\ell}+\sum_{j=1}^{M_{2}} z_{\ell} .
$$

Let $z_{l}:=\sum_{j=1}^{M_{2}} z_{\ell}^{j}$. Even though $z_{\ell}^{j}$ 's are not independent, any linear combination of $z_{l}^{j}$, s is Gaussian, therefore $z_{\ell}$ is $\mathcal{C} N\left(0, \sigma^{2}\right)$ distributed for some $\sigma^{2}$. Note that $\sigma^{2}$ depends on the channel coefficients, however, as shown in Theorem 2.3 of [24], $z_{\ell}$ is white in the scale of interest and without loss of generality $z_{\ell}$ can be modeled as $\mathcal{C} N(0,1)$, i.e., independent of channel coefficients in the outage analysis.
Let $\mathrm{SNR}:=\theta E$, then

$$
\begin{aligned}
& P_{\text {out }}(r \log \mathrm{SNR}) \\
= & P\left(\log \left(1+\mathrm{SNR} \sum_{j=1}^{M_{2}} \sum_{k=1}^{M_{1}}\left|g_{k j}\right|^{2}\left(\sum_{m=1}^{M_{0}}\left|h_{m k}\right|^{2}\right)\right) \leq r \log \mathrm{SNR}\right) \\
\leq & P\left(\sum_{k=1}^{M_{1}} \sum_{j=1}^{\min \left\{M_{0}, M_{2}\right\}}\left|g_{k j}\right|^{2}\left|h_{j k}\right|^{2} \leq \mathrm{SNR}^{-(1-r)}\right) \\
\leq & P\left(\max _{\left\{j=1, \ldots, \min \left\{M_{0}, M_{2}\right\}, k=1, \ldots, M_{1}\right\}}\left|g_{k j}\right|^{2}\left|h_{j k}\right|^{2} \leq \mathrm{SNR}^{-(1-r)}\right) .
\end{aligned}
$$

Since $\left|g_{k j}\right|^{2}\left|h_{j k}\right|^{2}$ are i.i.d. for $j=1, \ldots, \min \left\{M_{0}, M_{2}\right\}, k=$ $1, \ldots, M_{1}$ and the total number of terms are $\min \left\{M_{0} M_{1}, M_{1} M_{2}\right\}$,

$$
\begin{aligned}
& P_{\text {out }}(r \log \mathrm{SNR}) \\
\doteq & P\left(\left|g_{11}\right|^{2}\left|h_{11}\right|^{2} \leq \mathrm{SNR}^{-(1-r)}\right)^{\min \left\{M_{0} M_{1}, M_{1} M_{2}\right\}} .
\end{aligned}
$$

Note that $P\left(\left|g_{11}\right|^{2}\left|h_{11}\right|^{2} \leq \mathrm{SNR}^{-(1-r)}\right)$ is the outage probability of a single input single output system which can be computed easily using [29], and is given by

$$
P\left(\left|g_{11}\right|^{2}\left|h_{11}\right|^{2} \leq \mathrm{SNR}^{-(1-r)}\right) \doteq \mathrm{SNR}^{-(1-r)}, r \leq 1 .
$$

Thus, $P_{\text {out }}(r \log \mathrm{SNR}) \doteq \mathrm{SNR}^{-\min \left\{M_{0} M_{1}, M_{1} M_{2}\right\}(1-r)}$, $r \leq 1$, and we have that $d_{\text {out }}(r)=\min \left\{M_{0} M_{1}, M_{1} M_{2}\right\}$ $(1-r), r \leq 1$. Thus, the maximum diversity gain of the COSTBCs is $d_{\text {out }}(0)=\min \left\{M_{0} M_{1}, M_{1} M_{2}\right\}$ which equals the diversity gain upper bound (Theorem 1). Thus, we have shown that COSTBCs achieve the maximum diversity gain in a 2-hop network. Next, using induction, we prove the result for any $k$-hop network.

Assume that the result is true for a $k$-hop network $(k \geq 2)$, and we will prove that it is true for a $k+1$-hop network. Similar to 2-hop case, for a $k$-hop network also, at the destination, the received signal can be separated in terms of the individual constituent symbols of the OSTBC transmitted by the source. Thus, the received signal at the destination of the $k$-hop network corresponding to the signal $s_{\ell}$ can be written as

$$
y_{\ell}^{k}=\sqrt{\theta_{k-1} E_{k-1}} \sum_{i=1}^{M_{k}} c_{i} s_{\ell}+z_{\ell},
$$

where $\theta_{k-1}$ is the normalization constant so as to ensure the average power constraint of $E_{k-1}$ is satisfied at the relay stage $k-1, s_{\ell}$ is the $\ell$ th, $\ell=1,2, \ldots, L$ symbol transmitted from the source, $c_{i}$ is the channel gain experienced by $s_{\ell}$ at the $i$ th antenna of the destination, and $z_{l}$ is the AWGN with variance $\sigma_{k}^{2}$.

Now we extend the $k$-hop network to a $k+1$-hop network by assuming that the actual destination to be one more hop away and using the destination of the $k$-hop case as the $k$ th relay stage with $M_{k}$ relays by separating the $M_{k}$ antennas into $M_{k}$ relays with single antenna each. Similar 
to the $k$-hop case, the received signal at the destination of the $k+1$-hop can also be separated in terms of individual constituent symbols of the OSTBC transmitted by the source, which is given by

$$
y_{\ell}^{k+1}=\sqrt{\theta_{k} E_{k}} \sum_{i=1}^{M_{k}} c_{i}\left(\sum_{j=1}^{M_{k+1}}\left|g_{i j}\right|^{2}\right) s_{\ell}+n_{\ell}, \ell=1, \ldots, L,
$$

where $\theta_{k}$ is a constant to ensure the power constraint of $E_{k}$ in the $k+1$-hop network, $g_{i j}$ is the channel between the $i$ th relay of relay stage $k$ and the $j$ th antenna of the destination, and $n_{l}$ is the AWGN with variance $\sigma_{k+1}^{2}$. Let $y_{\ell}^{k+1}:=\sum_{i=1}^{M_{k}} y_{\ell i}$, and $q_{i}:=c_{i}\left(\sum_{j=1}^{M_{k+1}}\left|g_{i j}\right|^{2}\right)$, where

$$
y_{\ell i}=\sqrt{\theta_{k} E_{k}} q_{i} s_{\ell}+n_{\ell i}
$$

for each $\ell=1, \ldots, L$, where $n_{\ell i}=n_{\ell} / M_{k}$. Recall from induction hypothesis that the diversity gain of COSTBCs with channel $c_{i}, \forall i$ (15) is $\alpha:=$ $\min \left\{\min \left\{M_{n} M_{n+1}\right\}, M_{k-1}\right\}, n=0,1, \ldots, k-2$, by restricting the destination of the $k$-hop network to have only single antenna, and with channel $\sum_{i=1}^{M_{k}} c_{i}$ is $\min \left\{M_{n} M_{n+1}\right\}, n=0,1, \ldots, k-1$, respectively. Thus, if the diversity gain of COSTBCs with channel $q_{i}$ (17) is $\min \left\{\min \left\{M_{n} M_{n+1}\right\}, M_{k-1}, M_{k+1}\right\} n=0,1, \ldots, k-$ 2, then, since $\sum_{j=1}^{M_{k+1}}\left|g_{i j}\right|^{2}$ are independent $\forall i$, it follows that the diversity gain of COSTBCs with channel $\sum_{i=1}^{M_{k}} q_{i}$ is $\min \left\{M_{n} M_{n+1}\right\}, n=0,1, \ldots, k$. Next, we show that the diversity gain of COSTBCs with channel $q_{i}$ is $\min \left\{\min \left\{M_{n} M_{n+1}\right\}, M_{k-1}, M_{k+1}\right\}, n=0,1, \ldots, k-2$.

To compute the diversity gain of COSTBCs with channel $q_{i}$ (17), we use the outage probability formulation [29] as follows. Let $\sigma^{2}$ be the variance of $n_{\ell i}(17), \sigma^{2}=\frac{\sigma_{k+1}^{2}}{M_{k}^{2}}$, however, as before using Theorem 2.3 of [24] $n_{\ell i}$ is white in the scale of interest, and can be modeled as $\mathcal{C} N(0,1)$. Let $S N R:=\theta_{k} E_{k}$, then the outage probability of (17) is

$$
\begin{aligned}
& \text { Let } Z:=\sum_{j=1}^{M_{k+1}}\left|g_{i j}\right|^{2} \text {. Then } \\
& P_{\text {out }}(r \log \mathrm{SNR}) \leq P\left(Z \leq \mathrm{SNR}^{-(1-r)}\right) \\
& +\int_{\mathrm{SNR}^{-(1-r)}}^{\infty} \int_{0}^{\mathrm{SNR}^{-(1-r)} / z} f_{c_{i}}(y) d y f_{Z}(z) d z .
\end{aligned}
$$

By induction hypothesis, the diversity gain of COSTBCs with $c_{i}$ is $\alpha$, i.e.,

$$
\begin{aligned}
& P\left(c_{i} \leq \frac{\mathrm{SNR}^{-(1-r)}}{z}\right) \\
= & \int_{0}^{\mathrm{SNR}^{-(1-r)} / z} f_{c_{i}}(y) d y \leq k_{4}\left(\frac{\mathrm{SNR}^{-(1-r)}}{z}\right)^{\alpha}
\end{aligned}
$$

where $k_{4}$ is a constant. Thus,

$$
\begin{aligned}
P_{\text {out }}(r \log \mathrm{SNR}) \leq & P\left(Z \leq \mathrm{SNR}^{-(1-r)}\right) \\
& +\int_{\mathrm{SNR}^{-(1-r)}}^{\infty} k_{4} \mathrm{SNR}^{-\alpha(1-r)}\left(\frac{1}{z}\right)^{\alpha} f_{Z}(z) d z .
\end{aligned}
$$

Since $Z$ is a gamma distributed random variable with PDF $\frac{e^{-z} z^{M_{k+1}-1}}{M_{k+1}-1 !}$, the first term in $P_{\text {out }}(r \log$ SNR) expression can be found in [29] and is given by $P\left(Z \leq \mathrm{SNR}^{-(1-r)}\right) \doteq$ $\mathrm{SNR}^{-M_{k+1}(1-r)}$. Now we are left with computing the second term which can be done as follows.

$$
\begin{aligned}
& \int_{\mathrm{SNR}^{-(1-r)}}^{\infty} k_{4} \mathrm{SNR}^{-\alpha(1-r)}\left(\frac{1}{z}\right)^{\alpha} f_{Z}(z) d z \\
& \doteq k_{4} \mathrm{SNR}^{-\alpha(1-r)} \int_{\mathrm{SNR}^{-(1-r)}}^{\infty} z^{-\alpha} \frac{e^{-z} z^{M_{k+1}-1}}{M_{k+1}-1 !} d z \\
& \doteq \mathrm{SNR}^{-\alpha(1-r)} .
\end{aligned}
$$

Thus, from (18) it follows that

$$
\begin{aligned}
P_{\text {out }}(r \log \mathrm{SNR}) & \doteq \mathrm{SNR}^{-M_{k+1}(1-r)}+\mathrm{SNR}^{-\alpha(1-r)} \\
& \doteq \mathrm{SNR}^{-\min \left\{M_{k+1}, \alpha\right\}(1-r)} .
\end{aligned}
$$

Using the definition of diversity gain, it follows that the diversity gain of COSTBCs with channel $q_{i}$ is equal to $\min \left\{\alpha, M_{k+1}\right\}$, which implies that the diversity gain

$$
\begin{aligned}
P_{\text {out }}(r \log \mathrm{SNR}) & :=P\left(\log \left(1+\mathrm{SNR}_{i} \sum_{j=1}^{M_{k+1}}\left|g_{i j}\right|^{2} \leq r \log \mathrm{SNR}\right)\right) . \\
P_{\text {out }}(r \log \mathrm{SNR}) & \doteq P\left(\sum_{j=1}^{M_{k+1}}\left|g_{i j}\right|^{2} \leq \mathrm{SNR}^{-(1-r)}\right) P\left(c_{i} \sum_{j=1}^{M_{k+1}}\left|g_{i j}\right|^{2} \leq\left.\mathrm{SNR}^{-(1-r)}\left|\sum_{j=1}^{M_{k+1}}\right| g_{i j}\right|^{2} \leq \mathrm{SNR}^{-(1-r)}\right) \\
& +P\left(\sum_{j=1}^{M_{k+1}}\left|g_{i j}\right|^{2}>\mathrm{SNR}^{-(1-r)}\right) P\left(c_{i} \sum_{j=1}^{M_{k+1}}\left|g_{i j}\right|^{2} \leq\left.\mathrm{SNR}^{-(1-r)}\left|\sum_{j=1}^{M_{k+1}}\right| g_{i j}\right|^{2}>\mathrm{SNR}^{-(1-r)}\right) \\
& \leq P\left(\sum_{j=1}^{M_{k+1}}\left|g_{i j}\right|^{2} \leq \mathrm{SNR}^{-(1-r)}\right)+P\left(c_{i} \sum_{j=1}^{M_{k+1}}\left|g_{i j}\right|^{2} \leq\left.\mathrm{SNR}^{-(1-r)}\left|\sum_{j=1}^{M_{k+1}}\right| g_{i j}\right|^{2}>\mathrm{SNR}^{-(1-r)}\right) .
\end{aligned}
$$


of COSTBCs with the received signal model (16) is $\min \left\{\alpha M_{k}, M_{k} M_{k+1}\right\}$. Note that the upper bound on the diversity gain (Theorem 1) is also $\min \left\{\alpha M_{k}, M_{k} M_{k+1}\right\}$, and hence we conclude that the COSTBCs achieve the maximum diversity gain in an $N$-hop network.

\section{Appendix 2}

In this section, we prove that COSTBCs have the single symbol decodable property for $N=2$ and $M_{2}=1$.

Let $\mathbf{S}_{0}$ be the transmitted OSTBC from the source, and $\mathbf{s}=\left[s_{1}, \ldots, s_{L}\right]^{T}$ be the vector of the constituent symbols of $\mathbf{S}_{0}$. Then from (3), using CSI, the received signal $\mathbf{y}_{k}^{1}$ at the $k$ th relay of relay stage 1 can be transformed into $\hat{\mathbf{y}}_{k}^{1}$, where $\hat{\mathbf{y}}_{k}^{1}=\sqrt{E_{0} \mathrm{H}_{k}^{1}} \mathbf{s}+\hat{\mathbf{n}}_{k}^{1}$, where $\mathrm{H}_{k}^{1}$ is defined in (2), and the entries of $\hat{\mathbf{n}}_{k}$ are independent and $\mathcal{C} N(0,1)$ distributed. For $N=2$, and $M_{2}=1$, from (6), the received signal at the destination can be written as $\mathbf{y}_{1}^{2}=\left[\mathbf{t}_{1}^{1} \mathbf{t}_{2}^{1} \ldots \mathbf{t}_{M_{1}}^{1}\right] \mathbf{g}_{1}+\mathbf{z}_{1}^{2}$, where $\mathbf{t}_{k}^{1}$ is the transmitted vector from relay $k(4)$ of relay stage 1 . The received signal $\mathbf{y}_{1}^{2}$ can also be written as

$$
\begin{gathered}
\mathbf{y}_{1}^{2}=\sqrt{\frac{E_{0} E_{1} M}{L \gamma}} \mathbf{S}_{1} \underbrace{\left[\sqrt{\sum_{m=1}^{M_{0}}\left|h_{m 1}\right|^{2}} g_{11} \sqrt{\sum_{m=1}^{M_{0}}\left|h_{m 2}\right|^{2}} g_{21} \ldots \sqrt{\sum_{m=1}^{M_{0}}\left|h_{m M_{1}}\right|^{2}} g_{M_{1} 1}\right]^{T}}_{\mathbf{C}} \\
+\underbrace{\sqrt{\frac{E_{1} M_{1}}{L \gamma}}\left[\mathbf{A}_{1} \hat{n}_{1}+\mathbf{B}_{1} \hat{n}_{1}^{\dagger} \mathbf{A}_{2} \hat{n}_{2}+\mathbf{B}_{2} \hat{n}_{2}^{\dagger} \ldots \mathbf{A}_{M_{1}} \hat{n}_{M_{1}}+\mathbf{B}_{M_{1}} \hat{n}_{M_{1}}^{\dagger}\right] \mathbf{g}_{1}+\mathbf{z}_{1}^{2}}_{\mathbf{w}},
\end{gathered}
$$

where $\mathbf{S}_{1}=\left[\mathbf{A}_{1}^{1} \mathbf{s}+\mathbf{B}_{1}^{1} \mathbf{s}^{\dagger} \mathbf{A}_{2}^{1} \mathbf{s}+\mathbf{B}_{2}^{1} \mathbf{s}^{\dagger} \ldots \mathbf{A}_{M_{1}} \mathbf{s}+\mathbf{B}_{M_{1}} \mathbf{s}^{\dagger}\right]$

Let $\mathbf{R}=\mathbb{E}\left\{\mathbf{w w}^{*}\right\}$ be the noise covariance matrix.

Note that

$$
\mathbf{R}=\frac{E_{1} M_{1}}{L \gamma}\left[\begin{array}{cccc}
\mathbf{g}_{1}^{*} \mathbf{D}_{1} \mathbf{g}_{1} & 0 & \ldots & 0 \\
0 & \mathbf{g}_{1}^{*} \mathbf{D}_{2} \mathbf{g}_{1} & \ldots & 0 \\
0 & \ldots & \ddots & 0 \\
0 & \ldots & 0 & \mathbf{g}_{1}^{*} \mathbf{D}_{M_{1}} \mathbf{g}_{1}
\end{array}\right]+\mathbf{I}_{M_{1}}
$$

where $\mathbf{D}_{j}=\left(\mathbf{A}_{j}^{1} \mathbf{A}_{j}^{1 *}+\mathbf{B}_{j}^{1} \mathbf{B}_{j}^{1 *}\right)$ is a diagonal matrix whose diagonal entries are either zero or one since $\mathbf{A}_{j}$ and $\mathbf{B}_{j}$ are constituents of an OSTBC, and the number of ones in $\mathbf{D}_{j}$ is equal to $k$, if $\mathbf{A}_{j}$ and $\mathbf{B}_{j}$ are constituents of a rate $k / n$ OSTBC. Note that the locations of non-zero entries of $\mathbf{D}_{j}$ can be different for different $j$ 's, and hence $\mathbf{R}$ is not necessarily a scaled identity matrix. Therefore, because of the non-diagonal structure of $\mathbf{R}^{-1}$, the ML decoding metric (8) cannot be split in several terms, where each term is a function of only one of the constituent symbols of $\mathbf{S}_{1}$. Therefore in general, COSTBCs are not single symbol decodable. The problem can, however, be fixed easily by adding an additional channel coefficient-dependent noise vector to the received signal at the receiver as follows.

Let $\mathbf{1}_{j}=\operatorname{diag}\left(\mathbf{D}_{j}\right), \mathbf{1}_{j} \in\{0,1\}^{M_{1} \times 1}$, and $\mathbf{1}_{j}^{c}$ be the complement of $\mathbf{1}_{j}$, i.e., any entry of $\mathbf{1}_{j}^{c}$ is 1 if that entry of $\mathbf{1}_{j}$ is 0 , and vice versa. Let $\zeta_{j}=\mathbf{1}_{j}^{c}\left[\left|g_{11}\right|^{2}\left|g_{21}\right|^{2} \ldots\left|g_{M_{1} 1}\right|^{2}\right]^{T}$, and $\mathbf{a}=\sqrt{\frac{E_{1} M_{1}}{L \gamma}}\left[\sqrt{\zeta_{1}} u_{1} \sqrt{\zeta_{2}} u_{2} \ldots \sqrt{\zeta_{M_{1}}} u_{M_{1}}\right]^{T}$, where $u_{i}, i=1, \ldots, M_{1}$ are i.i.d. with $\mathcal{C} N(0,1)$ distribution. Then to make the noise covariance a scaled identity matrix, a can be added to the received signal at the destination $\mathbf{y}_{1}^{2}$. With $\mathbf{y}_{a}=\mathbf{y}_{1}^{2}+\mathbf{a}$, the noise vector $\mathbf{w}_{a}=\mathbf{w}+\mathbf{a}$, and the covariance matrix

$$
R_{\mathbf{w}_{a}}=\mathbb{E}\left\{\mathbf{n}_{a} \mathbf{n}_{a}^{*}\right\}=\frac{E_{1} M_{1}}{L \gamma}\left[\begin{array}{cccc}
\left|\mathbf{g}_{1}\right|^{2} & 0 & \ldots & 0 \\
0 & \left|\mathbf{g}_{1}\right|^{2} & \ldots & 0 \\
0 & \ldots & \ddots & 0 \\
0 & \ldots & 0 & \left|\mathbf{g}_{1}\right|^{2}
\end{array}\right]+\mathbf{I}_{M_{1}}
$$

which is a scaled identity matrix. Hence, using $\mathbf{y}_{a}$ (instead of $\mathbf{y}$ ) to decode $\mathbf{S}_{1}$, the ML decoding metric (8) splits in $L$ different terms, where each term is a function of only one of the constituent symbols of $\mathbf{S}_{0}$, and the COSTBC is single symbol decodable for $N=2$, and $M_{2}=1$. 


\section{Competing interests}

The authors declare that they have no competing interests.

\section{Acknowledgements}

This material is based upon work supported by the Army Research Laboratory Labs W91 1NF-10-1-0420 and by the National Science Foundation NSF-CCF-1218338.

\section{Author details}

${ }^{1}$ School of Technology and Computer Science, Tata Institute of Fundamental Research, Mumbai 400005, India. ${ }^{2}$ Wireless Networking and Communications Group, Department of Electrical and Computer Engineering, The University of Texas at Austin, 1 University Station C0803, Austin, TX 78712-0240, USA.

\section{Received: 20 November 2012 Accepted: 18 March 2013}

Published: 25 April 2013

\section{References}

1. VTarokh, H Jafarkhani, A Calderbank, Space-time block coding for wireless communications: performance results. IEEE J. Sel. Areas Commun. 17(3), 451-460 (1999)

2. J Laneman, G Wornell, Distributed space-time-coded protocols for exploiting cooperative diversity in wireless networks. IEEE Trans. Inf. Theory. 49(10), 2415-2425 (2003)

3. $Y$ Jing, B Hassibi, Diversity analysis of distributed space-time codes in relay networks with multiple transmit/receive antennas. EURASIP J. Adv. Signal Process. 2008, Article ID 254573 (2008)

4. R Nabar, H Bolcskei, F Kneubuhler, Fading relay channels: performance limits and space-time signal design. IEEE J. Sel. Areas Commun. 22(6), 1099-1109 (2004)

5. $\quad Y$ Jing, $B$ Hassibi, Distributed space-time coding in wireless relay networks. IEEE Trans. Wirel. Commun. 5(12), 3524-3536 (2006)

6. S Yang, J-C Belfiore, Optimal space time codes for the MIMO amplify-and-forward cooperative channel. IEEE Trans. Inf. Theory. 53(2), 647-663 (2007)

7. G Susinder Rajan, B Sundar Rajan, in Proceedings of IEEE Information Theory Workshop. A non-orthogonal distributed space-time coded protocol, Part-l: signal model and design criteria (IEEE, Punta del Este, Uruguay, October 22-26 2006), pp. 385-389

8. G Susinder Rajan, B Sundar Rajan, in Proceedings of IEEE Information Theory Workshop. A non-orthogonal distributed space-time coded protocol, Part-II: code construction and DM-G tradeoff (IEEE, Punta del Este, Uruguay, October 22-26 2006), pp. 488-492

9. F Oggier, B Hassibi, in IEEE International Symposium on Information Theory, 2006. An algebraic family of distributed space-time codes for wireless relay networks (IEEE, Punta del Este, Uruguay, July 2006), pp. 538-541

10. T Kiran, B Rajan, in IEEE International Symposium on Information Theory,2006. Distributed space-time codes with reduced decoding complexity, (July 2006), pp. 542-546

11. P Elia, P Vijay Kumar, Approximately universal optimality over several dynamic and non-dynamic cooperative diversity schemes for wireless networks, available at http://arxiv.org/pdf/cs.it/0512028, December 7, 2005

12. $Y$ Jing, $H$ Jafarkhani, Using orthogonal and quasi-orthogonal designs in wireless relay networks. IEEE Trans. Inf. Theory. 53(11), 4106-4118 (2007)

13. G Susinder Rajan, B Sundar Rajan, in IEEE International Symposium on Information Theory. Algebraic distributed space-time codes with low ML decoding complexity (IEEE, Nice, France, June 24-29 2007), pp. 1516-1520

14. Z Yi, I-M Kim, Joint optimization of relay-precoders and decoders with partial channel side information in cooperative networks. IEEE J. Sel. Areas Commun. 25(2), 447-458 (2007)

15. J Harshan, B Rajan, High-rate, single-symbol ML decodable precodedDSTBCs for cooperative networks. IEEE Trans. Inf. Theory. 55(5), 2004-2015 (2009)

16. J Harshan, B Rajan, A Hjorungnes, in IEEE International Symposium on Information Theory Proceedings (ISIT), 2010. Training-symbol embedded, high-rate complex orthogonal designs for relay networks (IEEE, Austin TX, USA, June 2010), pp. 2233-2237

17. D Sreedhar, A Chockalingam, B Rajan, in IEEE International Symposium on Information Theory, 2008. ISIT 2008. High-rate, single-symbol decodable distributed STBCs for partially-coherent cooperative networks, (July 2008), pp. 2538-2542

18. D Sreedhar, A Chockalingam, B Rajan, Single-symbol ML decodable distributed STBCs for partially-coherent cooperative networks. IEEE Trans. Wirel. Commun. 8(5), 2672-2681 (2009)

19. K Srinath, B Rajan, in IEEE Information Theory Workshop (ITW). Single real-symbol decodable, high-rate, distributed space-time block codes (IEEE, Dublin Ireland, 2010), pp. 1-5

20. T Duong, H Tran, in IEEE Radio and Wireless Symposium, 2008. Distributed space-time block codes with amicable orthogonal designs (IEEE, Orlando, FL, USA, January 2008), pp. 559-562

21. F Verde, A Scaglione, in IEEE International Conference on Acoustics Speech and Signal Processing (ICASSP). Randomized space-time block coding for distributed amplify-and-forward cooperative relays (IEEE, Dallas,TX, USA, 2010), pp. 3030-3033

22. F Oggier, B Hassibi, Code design for multihop wireless relay networks. EURASIP J. Adv. Signal Process. 2008, Article No. 68 (2008)

23. S Yang, J Belfiore, Diversity of MIMO multihop relay channels, available on http://arxiv.org/abs/0708.0386, August 2007

24. K Sreeram, S Birenjith, P Kumar, Dmt of multihop networks: end points and computational tools. IEEE Trans. Inf. Theory. 58(2), 804-819 (2012)

25. VTarokh, H Jafarkhani, A Calderbank, Space-time block codes from orthogonal designs. IEEE Trans. Inf. Theory. 45(5), 1456-1467 (1999)

26. S Alamouti, A simple transmit diversity technique for wireless communications. IEEE J. Sel. Areas Commun. 16(8), 1451-1458 (1998)

27. R Vaze, R Heath, in Proceedings of the Information Theory and Applications, 2008. Maximizing reliability in multi-hop wireless networks with cascaded space-time codes (IEEE, San Diego, CA, USA, January-February 2008)

28. R Vaze, R Heath, in IEEE International Symposium on Information Theory, 2008. ISIT 2008. Maximizing reliability in multi-hop wireless networks, (July 2008), pp. 11-15

29. L Zheng, D Tse, Diversity and multiplexing: a fundamental tradeoff in multiple-antenna channels. IEEE Trans. Inf. Theory. 49(5), 1073-1096 (2003)

30. B Sethuraman, B Rajan, V Shashidhar, Full-diversity, high-rate space-time block codes from division algebras. IEEE Trans. Inf. Theory. 49(10), 2596-2616 (2003)

31. KR Kumar, G Caire, Coding and decoding for the dynamic decode and forward relay protocol. IEEE Trans. Inf. Theory. 55(7), 3186-3205 (2009)

32. W Su, S Batalama, D Pados, On orthogonal space-time block codes and transceiver signal linearization. IEEE Commun. Lett. 10(2), 91-93 (2006)

33. W Su, X-G Xia, K Liu, A systematic design of high-rate complex orthogonal space-time block codes. IEEE Trans. Commun. 8(6), 380-382 (2004)

34. F Verde, in 5th International Symposium on Communications Control and Signal Processing (ISCCSP), 2012. Design of randomized space-time block codes for amplify-and-forward cooperative relaying (IEEE, Kyoto, Japan, May 2012), pp. 1-5

35. D Love, RW Heath Jr, T Strohmer, Grassmannian beamforming for multiple-input multiple-output wireless systems. IEEE Trans. Inf. Theory. 49(10), 2735-2747 (2003)

36. T Cover, J Thomas, Elements of Information Theory (Wiley, New York, 2004)

doi:10.1186/1687-1499-2013-113

Cite this article as: Vaze and Heath: Cascaded orthogonal space-time block codes for wireless multi-hop relay networks. EURASIP Journal on Wireless Communications and Networking 2013 2013:113. 